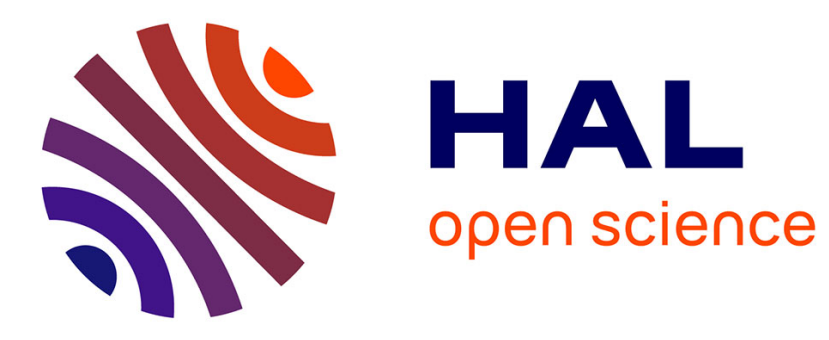

\title{
Frequency-Domain Stability Conditions for Asynchronously Sampled Decentralized LTI Systems
}

Jijju Thomas, Christophe Fiter, Laurentiu Hetel, Nathan van de Wouw, Jean-Pierre Richard

\section{- To cite this version:}

Jijju Thomas, Christophe Fiter, Laurentiu Hetel, Nathan van de Wouw, Jean-Pierre Richard. Frequency-Domain Stability Conditions for Asynchronously Sampled Decentralized LTI Systems. Automatica, In press, 129, 10.1016/j.automatica.2021.109603 . hal-03108717

\section{HAL Id: hal-03108717 https://inria.hal.science/hal-03108717}

Submitted on 13 Jan 2021

HAL is a multi-disciplinary open access archive for the deposit and dissemination of scientific research documents, whether they are published or not. The documents may come from teaching and research institutions in France or abroad, or from public or private research centers.
L'archive ouverte pluridisciplinaire $\mathbf{H A L}$, est destinée au dépôt et à la diffusion de documents scientifiques de niveau recherche, publiés ou non, émanant des établissements d'enseignement et de recherche français ou étrangers, des laboratoires publics ou privés. 


\title{
Frequency-Domain Stability Conditions for Asynchronously Sampled Decentralized LTI Systems
}

\author{
Jijju Thomas ${ }^{\text {a,b,c }}$, Christophe Fiter ${ }^{\text {a }}$, Laurentiu Hetel ${ }^{\text {a }}$, Nathan van de Wouw ${ }^{\text {c,d }}$, \\ Jean-Pierre Richard ${ }^{a, b}$ \\ ${ }^{\mathrm{a}}$ Univ. Lille, CNRS, Inria, Centrale Lille, UMR 9189 CRIStAL, F-59000 Lille, France \\ ${ }^{\mathrm{b}}$ Inria Lille, 40. av Halley, Villeneuve d'Ascq, France \\ ${ }^{\mathrm{c}}$ Eindhoven University of Technology, Eindhoven 5600 MB, The Netherlands \\ ${ }^{\mathrm{d}}$ Department of Civil, Environmental and Geo-Engineering, University of Minnesota, Minneapolis, MN 55455 USA
}

\begin{abstract}
This paper deals with the exponential stability analysis of decentralized, sampled-data, Linear Time Invariant (LTI) control systems with asynchronous sensors and actuators. We consider the case where each controller in the decentralized setting has its own sampling and actuation frequency, which translates to asynchrony between sensors and actuators. Additionally, asynchrony may be induced by delays between the sampling instants and actuation update instants as relevant in a networked context. The decentralized, asynchronous LTI system is represented as the feedback interconnection of a continuous-time LTI system operator and an operator that captures the effects of asynchrony induced by sampling and delay. By characterizing the properties of the operators using small-gain type Integral Quadratic Constraints (IQC), we provide criteria for exponential stability of the asynchronous, decentralized LTI state-space models. The approach provided in this paper considers two scenarios, namely the 'large-delay' case and the 'small-delay' case where the delays are larger and smaller than the sampling interval, respectively. The effectiveness of the proposed results is corroborated by a numerical example.
\end{abstract}

Key words: Sampled-data system, asynchronous sampling and actuation, decentralized system, integral quadratic constraints.

\section{Introduction}

Modern-day complex systems are hyper-connected with several wireless and wired components that interact with controllers and actuators. In such systems, due to the large number of distributed sensors and actuators, implementing a centralized control strategy is often not possible [2]. Decentralized control, wherein controllers are assigned to individual sub-systems, is often employed in such cases [1, 2]. Typical examples include Swarm Robotics, Vehicle Platooning, etc., [2, 21].

\footnotetext{
* This paper was not presented at any IFAC meeting. Corresponding author Jijju Thomas. Tel. +33-603763592.

Email addresses: j.thomas@tue.nl (Jijju Thomas), christophe.fiter@univ-lille.fr (Christophe Fiter), laurentiu.hetel@centralelille.fr (Laurentiu Hetel), nvd@tue.nl (Nathan van de Wouw),

jean-pierre.richard@centralelille.fr (Jean-Pierre Richard).
}

Implementing a decentralized control architecture provides certain advantages. Large-scale systems, the complexity of which prohibits a centralized controller design, are usually decoupled into subsystems. Consequently, the control design problem becomes a local problem, in the sense that global performance is achieved via local performance. Moreover, since the controllers are decoupled, diagnostics and maintenance tasks are easier. This results in overall lower running costs $[1,2]$.

Sensors and actuators in a decentralized scheme are typically deployed over aperiodic communication channels. However, local controllers are usually designed using classical sampled-data techniques [2]. This fact, in turn, poses a challenge in synchronization of different control system elements due to two main reasons. First, at an implementation level, individual controllers are usually algorithms programmed on embedded processors which work at different frequencies. Secondly, individual communication channels over which the sensor-actuator nodes are distributed, have unique network characteris- 


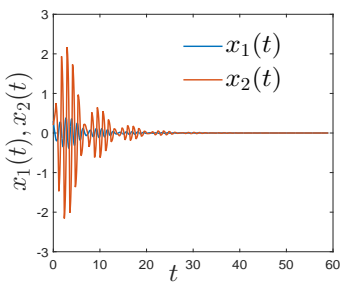

(a)

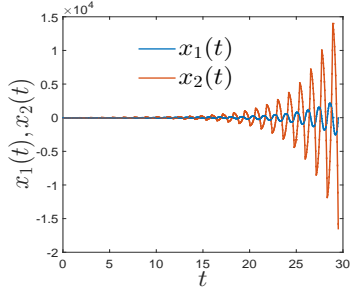

(b)
Fig. 1. (a) The decentralized LTI system (1) is stable for synchronous sampling with $T=0.59$. (b) Stability is lost when $x_{2}(t)$ is sampled asynchronously with respect to $x_{1}(t)$ with a shift of $\delta=0.2$.

tics such as communication delay, sampling and actuation frequencies, etc. The resulting asynchrony may in turn affect the overall performance of the system and even its stability. In this paper, this particular problem within the sampled-data implementation of decentralized controls is considered. In other words, we study the effect of asynchrony between local, possibly aperiodic, sampled-data controllers, on the overall stability of the system. The significance of such an analysis is corroborated using the following example studied in [24]. Consider the decentralized LTI system defined by

$$
\begin{array}{ll}
\Sigma_{1}: & \dot{x}_{1}(t)=-2 x_{1}(t)-x_{2}(t)+u_{1}(t) \\
\Sigma_{2}: & \dot{x}_{2}(t)=4 x_{2}(t)-2.8 x_{1}(t)+u_{2}(t),
\end{array}
$$

where $u_{1}(t)=-\hat{x}_{1}(t), u_{2}(t)=-4.6 \hat{x}_{2}(t)$ are the decentralized control inputs to systems $\Sigma_{1}$ and $\Sigma_{2}$, respectively, and $\hat{x}_{1}(t), \hat{x}_{2}(t)$ are the state values obtained through sampling and hold. In the event that both systems $\Sigma_{1}$ and $\Sigma_{2}$ are sampled periodically as well as synchronously with a sampling period $T=0.59$ (i.e., $\left.\hat{x}_{i}(t)=x_{i}(k T), \forall t \in[k T,(k+1) T), i=\{1,2\}\right)$, the overall system is asymptotically stable as illustrated in Figure 1a. However, as can be observed from Figure 1b, the stability is compromised when the sampling is periodic but control loops are asynchronous. Figure 1b presents the case when a shift $\delta=0.2$ is introduced in the sampling of the second state, i.e., when $\hat{x}_{2}(t)=x_{2}(k T+\delta), \forall t \epsilon$ $[k T+\delta,(k+1) T+\delta)$.

The stability problem can become even more complex when both the sensors and actuators involved within individual control loops are asynchronous. In this paper, we provide novel methods for the stability analysis of LTI systems with decentralized sampled-data linear controllers subject to asynchrony. The asynchrony in question is attributed to the separate sampling and actuation frequencies of each sensor and actuator node, as well as the delay induced in the control loop by control computation and communication latencies.

Mathematical problem settings that are closely related to the one considered in this paper, have previously been studied $[25,3,5,8]$. For example, in the case of central- ized controllers with aperiodic sampling and asynchrony between sensors and actuators, stability analysis methods have been proposed in [25]. However, the sampling and actuation frequencies were considered to be constant, and same for all sub-systems. In [3], $\mathcal{L}_{2}$-stability was analyzed for a distributed control system in which a single sensor transmits information to two distributed controllers with asynchronous actuation. The sampling and actuation scheme considered in [3] allows multiple samples to be overwritten before a hold update occurs. In comparison, we assume that actuation events are ordered. In [5], the asynchrony between sensors and actuators is controlled to attain desired levels of system performance, via decentralized event-triggered control. In this paper, we check the robustness of a decentralized system setting with respect to arbitrary asynchrony periods, implying that the time elapsed between sampling and actuation instants is arbitrary.

In literature, stability analysis of sampled-data systems are broadly classified into four approaches, namely the Time-delay, Discrete-time, Hybrid systems, and Inputoutput approaches. An overview of these approaches can be found in [12]. In this paper, we focus on the inputoutput approach [18], which was initially employed in the stability analysis of time-delay systems $[13,15,16$, 10]. The general idea of the approach, in the context of sampled-data systems, is to take into account the effects of sampling as perturbations and model it using operators. By studying these operators, powerful optimization-based stability conditions can be derived using Integral Quadratic Constraints (IQC) [18]. IQCs are inequalities that are used to exploit structural information about perturbations, characterize properties of external signals, etc. They offer a general framework for abstracting complex elements of dynamical system models (nonlinearities, delay, time-varying elements, etc.) to rigorously analyse robustness and performance using basic LTI models commonly employed in control engineering applications. The main advantage of an IQC-type formulation is its flexibility. Sampling is just one perturbation among others; the approach can be easily extended to take into account other performance and robustness specifications. In the case of LTI systems subject to aperiodic sampling or delay, the input-output approach leads to simple frequency-domain characterisations, which sometimes are model-free (measured frequency response functions) $[15,19]$.

The stability analysis of sampled-data systems using the input-output approach relies on two distinct formulations. In the first one, a purely operator-based formulation is considered, wherein the system is represented by operators with zero initial conditions $[15,19,3]$. This formulation leads to $\mathcal{L}_{2}$-stability conditions with respect to exogenous perturbations. Additionally, in this formulation, non-linearities can be treated as operators represented by perturbations. In the second type of formulation, state-space model representations with non-zero 
initial conditions are considered [20,7]. The robustness with respect to asynchrony is then given in terms of exponential stability property. Contrary to the first formulation, in this case, non-linear systems are handled by providing dissipativity type conditions on state-space models, using supply function characterisation of operators [20, 23]. Both formulations mentioned here may lead to similar stability conditions. Specifically, in the case of LTI systems, both formulations lead to characterisation in terms of IQC. More closely related to our problem formulation, IQC has previously been used in the $\mathcal{L}_{2}$ stability analysis of a single sensor-actuator system with aperiodic sampling $[19,11]$. In [10], it has been shown that $\mathcal{L}_{2}$-stability of a sampled-data system with respect to exogenous perturbations also implies asymptotic stability of the equilibrium of the state-space model. In [3], the $\mathcal{L}_{2}$-stability of a system in a distributed control setting, with asynchrony between sensors and actuators, is addressed. The result provided in [3], and earlier closely related works $[14,6]$, provide IQCs along with gain bound characterizations of the kind considered in this paper. Additionally, in [3], only boundedness of the system solutions with respect to exogenous perturbations was established. However, in [3, 4, 6, 14], richer characterizations that account for bounded gain, as well as passivity properties of the operator characterizing network effects, have been provided. The benefit of accounting for both gain bound and passivity properties has been illustrated in [4]. In the general case, there are no results in literature that provide relations between IQC formulations and exponential stability of asynchronous sampled-data control systems. In this paper, we bridge this gap in the case of LTI systems with decentralized controllers, by providing general exponential stability results based on IQC formulations.

The main contributions of this paper are as follows. The major result is an IQC-based framework for the exponential stability analysis of LTI state-space models of decentralized sampled-data (networked) systems with asynchrony. Novel aspects of this approach are detailed next. First, we propose a preliminary result introducing a novel and general framework for representing LTI state-space models of single-loop sampled-data systems with asynchronous sensors and actuators, in the robust control framework, as an interconnection between a continuous-time LTI system operator and an operator that captures the effects of asynchrony. Second, we apply this result to a generic class of LTI state-space models of aperiodically sampled asynchronous decentralized systems, and formulate small-gain type IQC conditions not only for $\mathcal{L}_{2}$-stability, but also for exponential stability. For the sake of generality, we consider the sampling and actuation intervals to be time-varying and possibly unknown (but bounded). Third, we consider two relevant scenarios, namely the large-delay and small-delay cases. As the name suggests, in the large-delay case, for individual control loops in the decentralized setting, the actuation corresponding to a measured (sampled) state could occur after the next sampling instant or instants. The only restriction is that the actuation instants for each control loop occurs in an order corresponding to the sampling instants. In this scenario, a single operator is used to capture the effects of asynchrony induced by sampling and delay. The second scenario, namely the small-delay case, implies that the actuation corresponding to a sampled state occurs before the next sampling instant. This scenario has been studied in numerous theoretical as well as practical settings (see [26, 27, 22]). For example, in [22], it was shown that in the case of a single sensor sampling periodically, when the sampled data experienced delays smaller than sampling-interval, the system was rendered unstable. The problem becomes much more complex when multiple systems are involved, with individual sensors and actuators having aperiodic, asynchronous sampling and actuation. For such scenarios, we provide a less conservative criterion in comparison to the criterion provided for the large-delay case, applied to the small-delay case. This is achieved by capturing the effects of sampling-induced asynchrony and delay-induced asynchrony, using two separate operators.

In a previous conference paper [24], we have used the Input-output approach to provide easy-to-check $\mathcal{L}_{2}$ stability conditions for a setting similar to the one considered in this paper. However, the results in [24] only established boundedness and did not take into account individual bounds on sampling and actuation frequencies, thereby leading to considerably more conservative results. Here, in this paper, we propose a novel approach that guarantees exponential stability of the state-space model by taking into account individual bounds on sampling and actuation frequencies, which ensures that the results in this paper are less conservative and more generic in comparison to the results in [24].

The remainder of this paper has been structured as follows. In Section 2, we provide a preliminary result that provides a general framework for representing LTI systems with asynchronous sensors and actuators, as an interconnection between a system operator and an operator capturing the effects of asynchrony. In Sections 3 and 4, we provide small-gain IQC-type stability conditions guaranteeing exponential stability of aperiodically sampled, decentralized, asynchronous LTI systems. In Section 3, the criterion is provided for the large-delay case, i.e., the transmission delay is larger than the sampling interval. In Section 4, a less conservative criterion is provided for the small-delay case, i.e., the transmission delay is smaller than the sampling interval. Section 5 provides a numerical example corroborating the results introduced in Sections 3 and 4.

Notations: $\mathbb{R}$ is the set of all real numbers, implying $\mathbb{R}^{n}$ is the set of all $n$-dimensional real vectors. $\mathbb{N}$ denotes the set of all natural numbers i.e., $\{0,1,2, \ldots, \infty\}$. The notation $\mathbb{N}^{\star}$ is used to denote the set $\{\mathbb{N} \backslash\{0\}\}$. $\operatorname{Diag}\left(M_{1}, M_{2}, \ldots, M_{n}\right)$ is the block-diagonal matrix with elements $M_{i}, i \in\{1,2, \ldots, n\}$, of appropriate dimensions. 
$\mathcal{L}_{2 e}(a, b)$ is the extended $\mathcal{L}_{2}$-space of all square integrable and Lebesgue measurable functions defined on the interval $[a, b]$, with the $\mathcal{L}_{2}$-norm defined as $\|q\|_{\mathcal{L}_{2}}^{2}=\langle q, q\rangle$, and the inner product $\langle p, q\rangle=\int_{a}^{b} p(s)^{T} q(s) d s$.

\section{Preliminary Result}

In this section, we provide a preliminary result on the remodelling of a generic single-loop, LTI system with asynchronous sensors and actuators, as the feedback interconnection of a continuous-time system operator and an operator that captures the effects of asynchrony. This result by itself bears significance in the robust control framework, wherein feedback interconnections of system operators are often considered. Consider the sampleddata LTI system defined by

$$
\begin{aligned}
\dot{x}(t) & =A x(t)+B K \hat{x}(t)+w(t), \\
x(0) & =x_{0},
\end{aligned}
$$

where $x \in \mathbb{R}^{n}, w \in \mathcal{L}_{2 e}^{n}[0, \infty)$ and

$$
\hat{x}(t)=\left\{\begin{array}{l}
x_{i n i t}, \forall t \in\left[0, a_{0}\right) \\
x\left(s_{k}\right), \forall t \in\left[a_{k}, a_{k+1}\right), k \in \mathbb{N}
\end{array}\right.
$$

with $x_{\text {init }} \in \mathbb{R}^{n}$ being some constant initial value applied at the actuator level. $A, B$ and $K$ are matrices of appropriate dimensions. The system under consideration follows from an LTI system controlled by state feedback over a sampled-data network with delay. The sampling sequence $\left\{s_{k}\right\}_{k \in \mathbb{N}}$ satisfying

$$
s_{k+1}=s_{k}+h_{k}, \forall k \in \mathbb{N}
$$

where the time-varying sampling interval $h_{k}$ satisfies $0<$ $h \leq h_{k} \leq \bar{h}, \forall k \in \mathbb{N}$. Similarly, the actuation sequence $\left.\overline{\{} a_{k}\right\}_{k \in \mathbb{N}}$ satisfies

$$
a_{k}=s_{k}+\tau_{k}, \forall k \in \mathbb{N},
$$

where $\tau_{k}$ represents the asynchrony (delay) between sampling and actuation instants and satisfies $0 \leq \tau \leq \tau_{k} \leq \bar{\tau}, \forall k \in \mathbb{N}$. In addition, the actuation instants satisfy

$$
a_{k} \leq a_{k+1}, \forall k \in \mathbb{N}
$$

Now consider the feedback interconnection of the form shown in Figure 2, where the dynamics of $\mathbf{G}$ are given by

$$
G:\left\{\begin{array}{l}
\dot{z}(t)=A_{c l} z(t)+B_{c l} u_{z}(t)+w(t) \\
y_{z}(t)=\dot{z}(t), z(0)=0, w(t) \in \mathcal{L}_{2 e}[0, \infty)
\end{array}\right.
$$

where $A_{c l}=A+B K, B_{c l}=B K, z \in \mathbb{R}^{n}$, and

$$
u_{z}(t)=e(t)+g(t)
$$

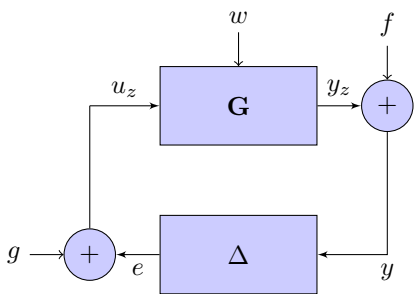

Fig. 2. The feedback interconnection of $\mathbf{G}$ and $\Delta$

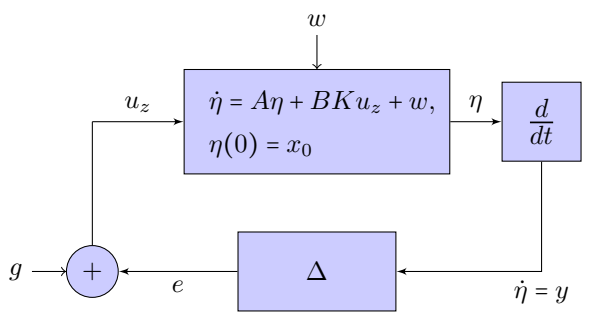

Fig. 3. The feedback-interconnection used as an intermediate while transitioning from system (2),(3) to system (7)-(10), as shown in proof of Theorem 1, in Appendix A.

with $g \in \mathcal{L}_{2 e}^{n}[0, \infty)$. The signal $e(t)$ is given by

$e(t)=(\Delta y)(t):=\left\{\begin{array}{l}0, \forall t \in\left[0, a_{0}\right) \\ -\int_{s_{k}}^{t} y(s) d s, \forall t \in\left[a_{k}, a_{k+1}\right), k \in \mathbb{N},\end{array}\right.$

where

$$
y=y_{z}+f,
$$

with $f \in \mathcal{L}_{2 e}[0, \infty)$. In the following theorem, we provide conditions under which the single-loop sampleddata LTI system (2)-(3) can be represented using the feedback-interconnection of operators $G$ and $\Delta$, given by (7) and (9), respectively. This theorem builds upon similar transformations given in $[3,6,11,14,15,16,19]$.

Theorem 1 Consider system (2),(3), the feedbackinterconnection (7)-(10), and sampling and actuation sequences satisfying (4) and (5), respectively. Consider

$$
\eta(t)=z(t)+e^{A_{c l} t} x_{0}
$$

where $z(t)$ follows the dynamics given by the interconnection (7)-(10), in which $f(t)=A_{c l} e^{A_{c l} t} x_{0}$ and

$$
g(t)=\left\{\begin{array}{l}
x_{\text {init }}-\mu(t), \forall t \in\left[0, a_{0}\right) \\
0, \forall t \geq a_{0},
\end{array}\right.
$$

with $\mu(t):\left[0, a_{0}\right) \mapsto \mathbb{R}^{n}$ given by $\mu(t)=e^{A t} x_{0}+$ $\left.\int_{0}^{t} e^{A(t-\tau)}\left(B K x_{\text {init }}+w(\tau)\right) d \tau\right)$. Then, for $x(t)$ given in (2), (3), we have $x(t)=\eta(t)=z(t)+e^{A_{c l} t} x_{0}$, for all $t \geq 0$.

Proof The proof is given in Appendix A. 


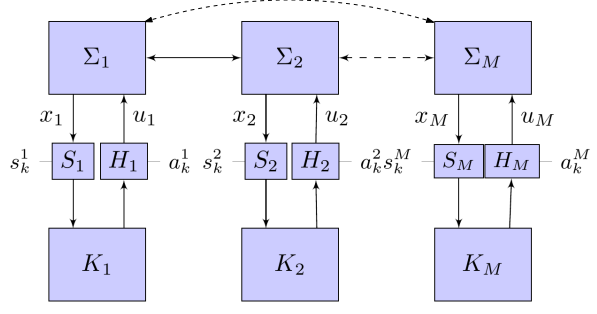

Fig. 4. A decentralized controller setup example. $S_{i}$ and $H_{i}$, for all $i \in\{1,2, \ldots, M\}$, denotes the sample and hold components, respectively, for the $i^{\text {th }}$ closed-loop.

Remark: The goal of Theorem 1 is to show that system (2), (3) (with non-zero initial conditions) can be represented in the form of system (7)-(10) (with zero initial conditions) for particular signals $f$ and $g$. To this end, we use an intermediate feedback-interconnection model given in Figure 3, in which system (2), (3) is represented as an interconnection between a nominal LTI system (with state variable $\eta$ ) with non-zero initial conditions, and an operator $\Delta$. This transformation is based on arguments previously used in $[3,6,11,14,15,16,19]$. Next, an additional transformation is used to relate the model in Figure 3 with the model (7)-(10), by deriving the appropriate signals $f$ and $g$ that represent the initial conditions.

Based on Theorem 1, in the following section, we will remodel a generic asynchronous, decentralized LTI system of the form shown in Figure 4, as a feedback interconnection of the form shown in Figure 2.

\section{Stability Analysis of Decentralized LTI Sys- tem: Large-delay Case}

In this section, we deal with the decentralized problem setting shown in Figure 4, in the large-delay case. First, we introduce the mathematical description of the problem setting. Constructive conditions are then given to analyse the stability of the decentralized setting.

\subsection{System description}

Consider the decentralized system configuration shown in Figure 4, wherein the dynamics of system $\Sigma_{i}$ is given by

$$
\dot{x}_{i}(t)=A_{i} x_{i}(t)+B_{i} u_{i}(t)+\sum_{j=1, i \neq j}^{M} A_{i j} x_{j}(t), \forall t \geq 0
$$

with $i \in\{1,2, . ., M\}, x_{i}(t) \in \mathbb{R}^{n_{i}}, x_{i}(0)=x_{0}^{i}$ and $u_{i}(t) \epsilon$ $\mathbb{R}^{m_{i}}$. The matrices $A_{i}, B_{i}$ and $A_{i j}$ are of appropriate dimensions. The term $A_{i j} x_{j}(t)$ denotes the influence of the states of the $j^{\text {th }}$ system $\Sigma_{j}$ on the dynamics of system $\Sigma_{i}$. Here, we consider the case where the control of the global system is linear. Furthermore, we assume

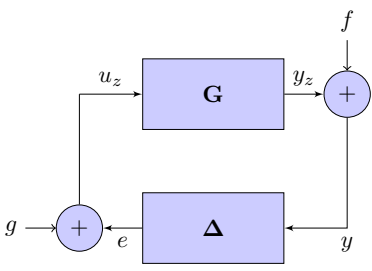

Fig. 5. Standard feedback interconnection of two exemplary operators $\mathbf{G}$ and $\boldsymbol{\Delta}$, which will be used to represent the decentralized system (13)-(16).

that it is decentralized in the sense that the control input $u_{i}(t)$ only depends on the local state variables $x_{i}(t)$. Furthermore, we consider that the control inputs are asynchronous. The system states $x_{i}(t)$ are sampled according to a sampling sequence $\left\{s_{k}^{i}\right\}_{k \in \mathbb{N}}$ defined by

$$
\left\{s_{k}^{i}: s_{k+1}^{i}-s_{k}^{i}=h_{k}^{i}, k \in \mathbb{N}, i \in\{1,2, . ., M\}\right\} .
$$

The sequence of sampling intervals $\left\{h_{k}^{i}\right\}_{k \in \mathbb{N}}$ satisfying $h_{k}^{i} \in\left[\underline{h}_{i}, \bar{h}_{i}\right]$ takes into account imperfection in sampling caused by, e.g., jitter, data packet dropouts, etc. Note that the sampling instants of different systems are not necessarily synchronous (hence the index $i$ in $s_{k}^{i}$ ). The control input $u_{i}(t)$ based on $x_{i}\left(s_{k}^{i}\right)$ will be implemented at a time instant $a_{k}^{i}$ at the level of the actuator of system $\Sigma_{i}$. We consider that the sequence of actuation times $\left\{a_{k}^{i}\right\}_{k \in \mathbb{N}}$ satisfies

$$
\left\{a_{k}^{i}: a_{k}^{i}=s_{k}^{i}+\eta_{k}^{i}, a_{k}^{i} \leq a_{k+1}^{i}, k \in \mathbb{N}, i \in\{1,2, \ldots, M\}\right\},
$$

where $\eta_{i}^{k} \in\left[\eta_{i}, \bar{\eta}_{i}\right]$ denotes the asynchrony between sensors and actuators. Such an asynchrony may be due to network delays, control computational delay, etc. Note that the constraint $a_{k}^{i} \leq a_{k+1}^{i}$ represents the large-delay case, wherein the network delay $\eta_{k}^{i}$ on the sampled state $x\left(s_{k}^{i}\right)$ can be greater than $h_{k}^{i}$, but the actuation instants stay ordered with respect to the sampling sequence. Without loss of generality, we consider $\bar{h}_{i}+\bar{\eta}_{i} \leq$ $a_{0}^{i} \leq a_{0}^{\star}$, where $a_{0}^{\star}=\max _{i=1}^{M}\left(\bar{h}_{i}+\bar{\eta}_{i}\right)$. Based on this consideration, the control input to the system $\Sigma_{i}$ is given by the sampled-data decentralized static state-feedback law

$$
u_{i}(t)=\left\{\begin{array}{l}
K_{i} x_{i n i t}^{i}, \forall t \in\left[0, a_{0}^{i}\right), \\
K_{i} x_{i}\left(s_{k}^{i}\right), \forall t \in\left[a_{k}^{i}, a_{k+1}^{i}\right), k \in \mathbb{N}
\end{array}\right.
$$

with some constant value $x_{i n i t}^{i} \in \mathbb{R}^{n_{i}}$. The main goal of this paper is to provide exponential stability criteria for the decentralized LTI system (13)-(16), uniformly with respect to the set of actuation and sampling instants.

\subsection{System reformulation}

Using the result in Theorem 1 as a stepping stone, we illustrate in this section how the decentralized system 
(13)-(16) can be represented by a feedback interconnection of the form given in Figure 5. This representation is useful in providing easy-to-check IQC-type stability criteria. The system (13)-(16) can also be given by

$$
\dot{x}(t)=A x(t)+B u(t), \forall t \geq 0,
$$

where

$$
\begin{aligned}
A & =\left[\begin{array}{cccc}
A_{1} & A_{12} & \ldots & A_{1 M} \\
A_{21} & A_{2} & \ldots & A_{2 M} \\
\vdots & \vdots & \ddots & \vdots \\
A_{M 1} & A_{M 2} & \ldots & A_{M}
\end{array}\right], \\
B & =\operatorname{diag}\left(B_{1}, B_{2}, \ldots, B_{M}\right)
\end{aligned}
$$

and $u(t)=\left[\begin{array}{lllll}u_{1}^{T}(t) & u_{2}^{T}(t) \ldots u_{M}^{T}(t)\end{array}\right]^{T}$, with $u_{i}(t)$ given by (16), for all $i \in\{1,2, \ldots, M\}$. Now consider a feedback interconnection $\mathbf{G}-\boldsymbol{\Delta}$ of the form given in Figure 5, where the operator $\mathbf{G}$ is defined by the dynamics

$$
\mathbf{G}:\left\{\begin{array}{l}
\dot{z}(t)=\tilde{A}_{c l} z(t)+B_{c l} u_{z}(t), \forall t \geq 0 \\
y_{z}(t)=\dot{z}(t)
\end{array}\right.
$$

with $z(t)=\left[z_{1}^{T}(t), z_{2}^{T}(t) \ldots z_{M}^{T}(t)\right]^{T}, z(0)=0$

$$
\tilde{A}_{c l}=\left[\begin{array}{cccc}
A_{c l}^{1} & A_{12} & \ldots & A_{1 M} \\
A_{21} & A_{c l}^{2} & \ldots & A_{2 M} \\
\vdots & \vdots & \ddots & \vdots \\
A_{M 1} & A_{M 2} & \ldots & A_{c l}^{M}
\end{array}\right]
$$

and

$$
B_{c l}=\operatorname{diag}\left(B_{1} K_{1}, B_{2} K_{2}, \ldots, B_{M} K_{M}\right),
$$

with $A_{c l}^{i}=A_{i}+B_{i} K_{i}, \forall i \in\{1,2, \ldots, M\}$, and $A_{i j}, A_{i}$, $B_{i}, K_{i}$ for all $j \in\{1,2, \ldots, M\}, j \neq i$ given by (13). Let the input $u_{z}$ be given by

$$
u_{z}(t)=e(t)+g(t)
$$

with $g \in \mathcal{L}_{2 e}^{n}[0, \infty)$, and the signal $e=\Delta y$ such that

$$
\Delta y=\left(\begin{array}{c}
\Delta_{1} y_{1} \\
\Delta_{2} y_{2} \\
\vdots \\
\Delta_{M} y_{M}
\end{array}\right)
$$

with $\Delta_{i}$ analogous to (9), for all $i \in\{1,2, \ldots, M\}$, and

$$
y=y_{z}+f,
$$

where $f \in \mathcal{L}_{2 e}^{n}[0, \infty)$. In the following theorem, we show how the system given by (13)-(16), can be remodelled as the feedback interconnection of $\mathbf{G}$ and $\boldsymbol{\Delta}$ given by (19)-(24), i.e.,

$$
\begin{aligned}
y & =\mathbf{G} u_{z}+f \\
u_{z} & =g+e, \\
e & =\boldsymbol{\Delta} y,
\end{aligned}
$$

by appropriately introducing the signals $f$ and $g$.

Theorem 2 Consider system (17), (16), the feedbackinterconnection (19)-(24), and the sampling and actuation sequences satisfying (14) and (15), respectively. Consider $\mu(t)=\left[\mu_{1}(t), \mu_{2}(t), \ldots, \mu_{M}(t)\right]$, where for all $i \in\{1,2, \ldots, M\}, \mu_{i}(t):\left[0, a_{0}^{\star}\right) \mapsto \mathbb{R}^{n}$ satisfies

$$
\dot{\mu}_{i}(t)=A_{i} \mu_{i}(t)+B_{i} u_{\mu}^{i}(t)+\sum_{j=1, i \neq j}^{M} A_{i j} \mu_{j}(t), \forall t \in\left[0, a_{0}^{\star}\right),
$$$$
\text { with } \mu_{i}(0)=x_{0}^{i}, a_{0}^{\star}=\max _{i=1}^{M}\left(a_{0}^{i}\right), \text { and }
$$

$$
u_{\mu}^{i}(t)=\left\{\begin{array}{l}
K_{i} x_{i n i t}^{i}, \forall t \in\left[0, a_{0}^{i}\right), \\
K_{i} \mu_{i}\left(s_{k}^{i}\right), \forall t \in\left[a_{k}^{i}, a_{k+1}^{i}\right) \cap\left[0, a_{0}^{\star}\right), k \in \mathbb{N},
\end{array}\right.
$$

with $x_{\text {init }}^{i} \in \mathbb{R}^{n_{i}}$, and

$$
\eta(t)=z(t)+\int_{0}^{t} e^{\tilde{A}_{c l}(t-\tau)} \tilde{A} e^{\hat{A} \tau} x_{0} d \tau+e^{\hat{A} t} x_{0}
$$

for all $t \geq 0$, where $z(t)$ is given by the dynamics (19)(24), $\tilde{A}_{c l}$ is given by (20),

$$
\hat{A}=\left[\begin{array}{cccc}
A_{c l}^{1} & 0 & \ldots & 0 \\
0 & A_{c l}^{2} & \ldots & 0 \\
\vdots & \vdots & \ddots & \vdots \\
0 & 0 & \ldots & A_{c l}^{M}
\end{array}\right]
$$

and $\tilde{A}=\tilde{A}_{c l}-\hat{A}$. Then, for

$$
f(t)=\tilde{A}_{c l} e^{\hat{A} t} x_{0}
$$

$$
\begin{gathered}
\text { and } g(t)=\left[g_{1}^{T}(t) g_{2}^{T}(t) \ldots g_{M}^{T}(t)\right]^{T}, \text { defined by } \\
g_{i}(t)=\left\{\begin{array}{l}
x_{\text {init }}^{i}-\mu_{i}(t), \forall t \in\left[0, a_{0}^{i}\right) \\
0, \forall t \geq a_{0}^{i}
\end{array}\right.
\end{gathered}
$$

we have that $x(t)=\eta(t)$ for all $t \geq 0$, where $x(t)$ is given by (17),(16).

Proof Consider the $i^{t h}$ closed-loop $\Sigma_{i}-K_{i}$ in Figure 4, given by (13)-(16). We have

$$
\dot{x}_{i}(t)=A_{i} x_{i}(t)+B_{i} u_{i}(t)+w_{i}(t), \forall t \geq 0
$$




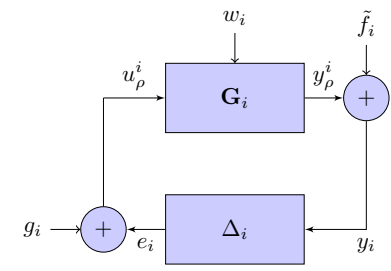

Fig. 6. The feedback interconnection of $\mathbf{G}_{\mathbf{i}}$ and $\Delta_{i}$, representing the $i^{t h}$ closed-loop $\Sigma_{i}-K_{i}$.

for all $i \in\{1,2, \ldots, M\}$, where

$$
w_{i}(t)=\sum_{j=1, i \neq j}^{M} A_{i j} x_{j}(t)
$$

By applying Theorem 1 , the $i^{\text {th }}$ closed-loop $\Sigma_{i}-K_{i}$ can be remodelled as the feedback interconnection $\mathbf{G}_{\mathbf{i}}-\Delta_{i}$ shown in Figure 6, where the operator $\Delta_{i}$ is given in a similar manner as defined in (9). The dynamics of system operator $\mathbf{G}_{\mathbf{i}}$ will be given by

$$
\begin{aligned}
& \dot{\rho}_{i}(t)=A_{c l}^{i} \rho_{i}(t)+B_{c l}^{i} u_{\rho}^{i}(t)+w_{i}(t), \forall t \geq 0, \\
& y_{\rho}^{i}(t)=\dot{\rho}_{i}(t), \rho_{i}(0)=0, i \in\{1,2, \ldots, M\} \\
& A_{c l}^{i}=A_{i}+B_{i} K_{i}, B_{c l}^{i}=B_{i} K_{i} .
\end{aligned}
$$

Also, the signals

$$
\tilde{f}_{i}(t)=A_{c l}^{i} e^{A_{c l}^{i} t} x_{0}^{i}
$$

and $g_{i}(t)=\left\{\begin{array}{l}x_{i n i t}^{i}-\mu_{i}(t), \forall t \in\left[0, a_{0}^{i}\right) \\ 0, \forall t \geq a_{0}^{i}\end{array}\right.$, , where $\mu_{i}(t)$ is generated by $(26),(27)$. Then, as a direct application of Theorem 1, for all $i \in\{1,2, \ldots, M\}$, the dynamics of the $i^{\text {th }}$ closed-loop $\Sigma_{i}-K_{i}$ is given by

$$
x_{i}(t)=\eta_{i}(t)=\rho_{i}(t)+e^{A_{c l}^{i} t} x_{0}^{i}, \forall t \geq 0,
$$

and consequentially, from (33), we have $w_{i}(t)=$ $\sum_{j=1, i \neq j}^{M} A_{i j}\left(\rho_{j}(t)+e^{A_{c l}^{j} t} x_{0}^{j}\right)$. Therefore, (34) gives

$$
\begin{aligned}
\dot{\rho}_{i}(t)= & A_{c l}^{i} \rho_{i}(t)+\sum_{j=1, i \neq j}^{M} A_{i j} \rho_{j}(t) \\
& +B_{c l}^{i} u_{\rho}^{i}(t)+\sum_{j=1, i \neq j}^{M} A_{i j} e^{A_{c l}^{j}} x_{0}^{j}
\end{aligned}
$$

for all $i \in\{1,2, \ldots, M\}$. From Figure 6 , for the $i^{\text {th }}$ feedback interconnection $\mathbf{G}_{\mathbf{i}}-\Delta_{i}$, we have $u_{\rho}^{i}(t)=e_{i}(t)+$ $g_{i}(t)$, where $g_{i}(t)$ is given by $(31)$ and $e_{i}(t)$ is given using the operator $\Delta_{i}$, defined similarly as in (9), i.e.,

$e_{i}(t)=\left(\Delta_{i} y_{i}\right)(t):=\left\{\begin{array}{l}0, \forall t \in\left[0, a_{0}^{i}\right) \\ -\int_{s_{k}^{i}}^{t} y_{i}(s) d s, \forall t \in\left[a_{k}^{i}, a_{k+1}^{i}\right), k \in \mathbb{N} .\end{array}\right.$ for all $i \in\{1,2, \ldots, M\}$. Additionally, for the $i^{\text {th }}$ feedback interconnection $\mathbf{G}_{\mathbf{i}}-\Delta_{i}$ shown in Figure 6 , we have

$$
y_{i}(t)=y_{\rho}^{i}(t)+\tilde{f}_{i}(t)
$$

where $y_{\rho}^{i}(t)=\dot{\rho}_{i}(t)$, and $\tilde{f}_{i}(t)$ is given by $(35)$.

Now, considering (36) for all $i \in\{1,2, \ldots, M\}$, we have

$$
\eta(t)=\rho(t)+e^{\hat{A} t} x_{0},
$$

where $\eta(t)=\left[\eta_{1}^{T}(t) \eta_{2}^{T}(t) \ldots \eta_{M}^{T}(t)\right]^{T}$, and $\hat{A}$ is given by (29). From (37), for all $i \in\{1,2, \ldots, M\}, \rho(t)$ is given by the dynamics of the system

$$
\begin{aligned}
\dot{\rho}(t) & =\tilde{A}_{c l} \rho(t)+B_{c l} u_{\rho}(t)+\tilde{A} e^{\hat{A} t} x_{0}, \\
y_{\rho}(t) & =\dot{\rho}(t), \rho(0)=0
\end{aligned}
$$

where

$$
\begin{aligned}
& \tilde{A}=\left[\begin{array}{cccc}
0 & A_{12} & \ldots & A_{1 M} \\
A_{21} & 0 & \ldots & A_{2 M} \\
\vdots & \vdots & \ddots & \vdots \\
A_{M 1} & A_{M 2} & \ldots & 0
\end{array}\right] \\
& \rho(t)=\left[\begin{array}{lllll}
\rho_{1}^{T}(t) & \rho_{2}^{T}(t) & \ldots & \rho_{M}^{T}(t)
\end{array}\right]^{T}, \\
& y_{\rho}(t)=\left[\begin{array}{llll}
\left(y_{\rho}^{1}\right)^{T}(t) & \left(y_{\rho}^{2}\right)^{T}(t) & \ldots & \left(y_{\rho}^{M}\right)^{T}(t)
\end{array}\right]^{T}, \\
& u_{\rho}(t)=\left[\begin{array}{llll}
\left(u_{\rho}^{1}\right)^{T}(t) & \left(u_{\rho}^{2}\right)^{T}(t) & \ldots & \left(u_{\rho}^{M}\right)^{T}(t)
\end{array}\right]^{T} \text {, } \\
& x_{0}=\left[\begin{array}{llll}
\left(x_{0}^{1}\right)^{T} & \left(x_{0}^{2}\right)^{T} \ldots\left(x_{0}^{M}\right)^{T}
\end{array}\right]^{T} .
\end{aligned}
$$

In (41), the matrices $\tilde{A}_{c l}, B_{c l}$ and $\hat{A}$ are given by (20), (21), and (29), respectively. Moreover, from (36) and (40), the state evolution of the dynamics of the decentralized system (13)-(16) is given by

$$
x(t)=\eta(t), \forall t \geq 0 .
$$

Additionally,

$$
u_{\rho}(t)=e(t)+g(t)
$$

where $g(t)=\left[\begin{array}{lllll}g_{1}^{T}(t) & g_{2}^{T}(t) & \ldots & g_{M}^{T}(t)\end{array}\right]^{T}$ and

$$
e(t)=\left[\begin{array}{llll}
e_{1}^{T}(t) & e_{2}^{T}(t) & \ldots & e_{M}^{T}(t)
\end{array}\right]^{T}=(\Delta y)(t),
$$

with $\Delta$ given by $(23), y(t)=\left[\begin{array}{llll}y_{1}^{T}(t) & y_{2}^{T}(t) & \ldots & y_{M}^{T}(t)\end{array}\right]^{T}$. From (39), we have

$$
y(t)=y_{\rho}(t)+\tilde{f}(t)
$$

where $y_{\rho}(t)$ is given by (43), and from $(35), \tilde{f}(t)=$ $\left[\begin{array}{lllll}\tilde{f}_{1}^{T}(t) & \tilde{f}_{2}^{T}(t) & \ldots & \tilde{f}_{M}^{T}(t)\end{array}\right]^{T}=\hat{A} e^{\hat{A} t} x_{0}$. Therefore, the de- 


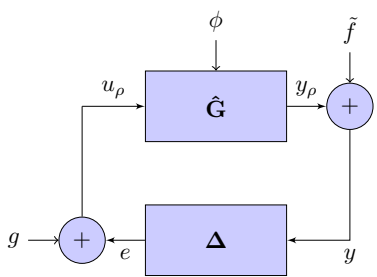

Fig. 7. The feedback interconnection of $\hat{\mathbf{G}}$ and $\boldsymbol{\Delta}$, representing the decentralized system (13)-(16).

centralized system given by (13)-(16), can be represented by the feedback interconnection (41), (45), (46), and (47), as shown in Figure 7, where $\hat{\mathbf{G}}$ describes the dynamics given by $(41)$ and $\phi(t)=\tilde{A} e^{\hat{A} t} x_{0}$. Now, consider the system defined by

$$
\begin{aligned}
\dot{z}(t) & =\tilde{A}_{c l} z(t)+B_{c l} u_{z}(t), \\
y_{z}(t) & =\dot{z}(t), z(0)=0,
\end{aligned}
$$

with $u_{z}(t)=u_{\rho}(t)$. Then, we have

$$
z(t)=\int_{0}^{t} e^{\tilde{A}_{c l}(t-\tau)} B_{c l} u_{z}(\tau) d \tau, \forall t \geq 0 .
$$

Similarly, from (41), we have

$$
\rho(t)=\int_{0}^{t} e^{\tilde{A}_{c l}(t-\tau)} B_{c l} u_{\rho}(\tau) d \tau+\int_{0}^{t} e^{\tilde{A}_{c l}(t-\tau)} \phi(\tau) d \tau .
$$

Since $u_{z}(t)=u_{\rho}(t)$, using (49) and (50), we have $\rho(t)=z(t)+\int_{0}^{t} e^{\tilde{A}_{c l}(t-\tau)} \phi(\tau) d \tau$. Consequently, we have $\dot{\rho}(t)=\dot{z}(t)+\phi(t)+\tilde{A}_{c l} \int_{0}^{t} e^{\tilde{A}_{c l}(t-\tau)} \phi(\tau) d \tau$. Therefore, we have $y_{\rho}(t)=y_{z}(t)+\hat{f}(t)$, where $\hat{f}(t)=\tilde{A} e^{\hat{A} t} x_{0}+\tilde{A}_{c l} \int_{0}^{t} e^{\tilde{A}_{c l}(t-\tau)} \tilde{A} e^{\hat{A} \tau} x_{0} d \tau$. Consequently, from (47), we have

$$
y(t)=y_{z}(t)+\hat{f}(t)+\tilde{f}(t)=y_{z}(t)+f(t)
$$

where $f(t)=\hat{f}(t)+\tilde{f}(t)$, i.e.,

$$
\begin{aligned}
f(t) & =(\tilde{A}+\hat{A}) e^{\hat{A} t} x_{0}+\tilde{A}_{c l} \int_{0}^{t} e^{\tilde{A}_{c l}(t-\tau)} \tilde{A} e^{\hat{A} \tau} x_{0} d \tau \\
& =\tilde{A}_{c l} e^{\hat{A} t} x_{0}+\tilde{A}_{c l} \int_{0}^{t} e^{\tilde{A}_{c l}(t-\tau)} \tilde{A} e^{\hat{A} \tau} x_{0} d \tau
\end{aligned}
$$

Therefore, the feedback interconnection in Figure 7, can also be expressed as the feedback interconnection shown in Figure 5, given by $y=\mathbf{G} u_{z}+f, u_{z}=g+e, e=\boldsymbol{\Delta} y$, where the system operator $\mathbf{G}$ is defined by the transfer function of the system given by (48), i.e., $\mathbf{G}(s)=\tilde{A}_{c l}\left(s I-\tilde{A}_{c l}\right)^{-1} B_{c l}+B_{c l}$. Also, from (44) and (40), we obtain $x(t)=\eta(t)=\rho(t)+e^{\hat{A} t} x_{0}=$ $z(t)+\int_{0}^{t} e^{\tilde{A}_{c l}(t-\tau)} \tilde{A} e^{\hat{A} \tau} x_{0} d \tau+e^{\hat{A} t} x_{0}$. Differentiating both sides of (44), and recalling that $\tilde{A}_{c l}=\tilde{A}+\hat{A}$, we have $\dot{x}(t)=\dot{z}(t)+\frac{d}{d t}\left(e^{\tilde{A}_{c l} t} \int_{0}^{t} e^{-\tilde{A}_{c l} \tau} \tilde{A} e^{\hat{A} \tau} x_{0} d \tau\right)+\hat{A} e^{\hat{A} t} x_{0}=$ $y_{z}(t)+\tilde{A}_{c l} e^{\hat{A} t} x_{0}+\tilde{A}_{c l} \int_{0}^{t} e^{\tilde{A}_{c l}(t-\tau)} \tilde{A} e^{\hat{A} \tau} x_{0} d \tau$. Therefore, from the definition of $f(t)$ in (52), and (51), we have

$$
\dot{x}(t)=y_{z}(t)+f(t)=y(t) .
$$

Remark: In the aforementioned theorem, it has to be noted that the operator $\mathbf{G}$ is based on a system with zero initial condition. The signals $\mu_{i}(t), f(t)$ and $g(t)$ are merely used for replicating the evolution of the decentralized LTI system with respect to the initial condition. This in turn leads to the construction of the signal $g_{i}(t)$ that serves as an input to the feedback interconnection of $\mathbf{G}$ and $\boldsymbol{\Delta}$ given in Figure 5 .

We have illustrated how the decentralized LTI system can be represented by the feedback interconnection of a system operator $\mathbf{G}$, and an operator $\boldsymbol{\Delta}$ that captures the effects of asynchrony. Feedback interconnections of this form, shown in Figure 5, are often studied in the robust control framework. This representation aids in providing simple $\mathcal{L}_{2}$-stability criteria as we have shown in [24]. In the next section, we illustrate the implication of such $\mathcal{L}_{2}$-stability properties on the exponential stability of the decentralized LTI system (13)-(16).

\subsection{Exponential stability criteria}

Typically, by obtaining bounds on the operator $\boldsymbol{\Delta}$, results that establish $\mathcal{L}_{2}$-stability properties of the feedback-interconnection $\mathbf{G}-\boldsymbol{\Delta}$ are obtained [24]. However, in the following theorem, we provide a result that establishes exponential stability of the system (13)(16), based on boundedness properties of the feedback interconnection $\mathbf{G}-\boldsymbol{\Delta}$ shown in Figure 5 .

Theorem 3 Suppose that $\tilde{A}_{c l}, \hat{A}$ given by (20), (29), respectively, are Hurwitz. Then, the decentralized system (13)-(16) is globally exponentially stable if the feedback interconnection $\mathbf{G}-\Delta$ defined by $(25)$ is $\mathcal{L}_{2}$-stable.

Proof The main idea of this proof is to use the particular signals $f$ and $g$ that established the equivalence, as proven in Theorem 2, between the decentralized LTI system (17), (16) and the feedback interconnection G$\boldsymbol{\Delta}$ given by (25), to prove exponential stability. First, we shall compute bounds on $f$ and $g$ to show that $f, g \in \mathcal{L}_{2 e}^{n}$. Boundedness of $x(t)$ : Recalling the definition of $f(t)$ in (30), we have,

$$
f(t)=\tilde{A}_{c l} e^{\hat{A} t} x_{0}+\tilde{A}_{c l} \int_{0}^{t} e^{\tilde{A}_{c l}(t-\tau)} \tilde{A} e^{\hat{A} \tau} x_{0} d \tau .
$$


Therefore,

$\|f(t)\| \leq\left(\left\|\tilde{A}_{c l} e^{\hat{A} t}\right\|+\left\|\tilde{A}_{c l} \int_{0}^{t} e^{\tilde{A}_{c l}(t-\tau)} \tilde{A} e^{\hat{A} \tau} d \tau\right\|\right)\left\|x_{0}\right\|$, where $\|\cdot\|$ denotes the Euclidean norm, and is given by $\|f(t)\|=\sqrt{f^{T}(t) f(t)}$. Since $\hat{A}$ and $\tilde{A}_{c l}$ are Hurwitz, there exist constants $c_{1}, c_{2}, \alpha_{1}<0$ and $\alpha_{2}<0$ such that $\left\|e^{\hat{A} t}\right\| \leq c_{1} e^{\alpha_{1} t},\left\|e^{\tilde{A}_{c l} t}\right\| \leq c_{2} e^{\alpha_{2} t}, \forall t \geq 0$. Consequently,

$$
\begin{aligned}
\|f(t)\| \leq & \left(\left\|\tilde{A}_{c l}\right\| c_{1} e^{\alpha_{1} t}\right. \\
& \left.+\|\tilde{A}\|\left\|\tilde{A}_{c l}\right\| c_{1} c_{2} \int_{0}^{t} e^{\alpha_{2}(t-\tau)} e^{\alpha_{1} \tau} d \tau\right)\left\|x_{0}\right\| \\
& =\left(\left\|\tilde{A}_{c l}\right\| c_{1} e^{\alpha_{1} t}\right. \\
& \left.\quad\|\tilde{A}\|\left\|\tilde{A}_{c l}\right\| c_{1} c_{2} e^{\alpha_{2} t} \int_{0}^{t} e^{\left(\alpha_{1}-\alpha_{2}\right) \tau} d \tau\right)\left\|x_{0}\right\| .
\end{aligned}
$$

Since $\int_{0}^{t} e^{\left(\alpha_{1}-\alpha_{2}\right) t} d \tau=\left\{\begin{array}{l}\frac{1}{\alpha_{1}-\alpha_{2}}\left(e^{\left(\alpha_{1}-\alpha_{2}\right) t}-1\right), \text { if } \alpha_{1} \neq \alpha_{2}, \\ t, \text { if } \alpha_{1}=\alpha_{2},\end{array}\right.$ we can state that $\|f(t)\| \leq\left(c_{3} e^{\alpha_{1} t}+c_{4} \zeta(t)\right)\left\|x_{0}\right\|$, where $c_{3}=\left\|\tilde{A}_{c l}\right\| c_{1}, c_{4}=\|\tilde{A}\|\left\|\tilde{A}_{c l}\right\| c_{1} c_{2}$, and $\zeta(t)=$ $\left\{\frac{1}{\alpha_{1}-\alpha_{2}}\left(e^{\alpha_{1} t}-e^{\alpha_{2} t}\right)\right.$, if $\alpha_{1} \neq \alpha_{2}$, $\left\{e^{\alpha_{2} t}\right.$, if $\alpha_{1}=\alpha_{2}$.

This implies that $\|f(t)\|$ can be upper-bounded by an exponentially decaying signal, i.e., $\lim _{t \rightarrow \infty} f(t) \rightarrow 0$ and also, $f \in \mathcal{L}_{2 e}^{n}[0, \infty)$. Now, recalling the definition of $g(t)$ in (31), and from (26), since $\mu(t)$ is bounded for all $t \in\left[0, a_{0}^{\star}\right)$ owing to its linearity, we have that $g(t)$ is bounded for all $t \in\left[0, a_{0}^{\star}\right)$ and $g(t)=0, \forall t \geq a_{0}^{\star}$, with $a_{0}^{\star}=\max _{i=1}^{M} a_{0}^{i}$. Consequentially, $\int_{0}^{\infty} g^{T}(t) g(t) d t=\mathcal{C}_{2}<\infty$, implying that $g \in \mathcal{L}_{2 e}^{n}[0, \infty)$. Since the feedback interconnection (25) is $\mathcal{L}_{2}$-stable, i.e., the mapping $\left[\begin{array}{l}f \\ g\end{array}\right] \mapsto\left[\begin{array}{c}y \\ u_{z}\end{array}\right]$ is $\mathcal{L}_{2}$-stable, we have $\int_{0}^{\infty}\left(y^{T}(\theta) y(\theta)+u_{z}^{T}(\theta) u_{z}(\theta)\right) d \theta \leq$ $\mathcal{C} \int_{0}^{\infty}\left(f^{T}(\theta) f(\theta)+g^{T}(\theta) g(\theta)\right) d \theta<\infty$, where the constant $\mathcal{C}>0$, implying that $y, u_{z} \in \mathcal{L}_{2 e}^{n}$. We know from the definition of the feedback interconnection (25), that $y_{z}=y-f$, implying that since $y, f \in \mathcal{L}_{2 e}^{n}$, we have $y_{z} \in \mathcal{L}_{2 e}^{n}$. As per definition of system operator $\mathbf{G}$ in (19), we have $y_{z}(t)=\dot{z}(t)=\tilde{A}_{c l} z(t)+B_{c l} u_{z}(t), \forall t \geq 0$. Therefore, $\tilde{A}_{c l} z(t)=y_{z}(t)-B_{c l} u_{z}(t)$, and since $\tilde{A}_{c l}$ is invertible, we have $z \in \mathcal{L}_{2 e}^{n}$. Recalling Theorem 2 , from (28), we have

$$
x(t)=z(t)+\int_{0}^{t} e^{\tilde{A}_{c l}(t-\tau)} \tilde{A} e^{\hat{A} \tau} x_{0} d \tau+e^{\hat{A} t} x_{0} .
$$

We know that $\hat{A}$ is Hurwitz implying $\lim _{t \rightarrow \infty} e^{\hat{A} t} x_{0} \rightarrow 0$. Additionally, the signal $\int_{0}^{t} e^{\tilde{A}_{c l}(t-\tau)} \tilde{A} e^{\hat{A} \tau} x_{0} d \tau \in \mathcal{L}_{2 e}^{n}$, since it is the response of a stable LTI system to the input $\tilde{A} e^{\hat{A} \tau} x_{0} \in \mathcal{L}_{2 e}^{n}$. Finally, since we have shown that $z \in \mathcal{L}_{2 e}^{n}$, from (56), we have $x \in \mathcal{L}_{2 e}^{n}$.

Exponential stability of $x(t)$ : From Theorem 2, we know that the feedback interconnection $\mathbf{G}-\boldsymbol{\Delta}$ defined by (25) represents the decentralized system (13)-(16), given by the homogenous state-space equation

$$
\dot{x}(t)=A x(t)+B K \hat{x}(t), x(0)=x_{0},
$$

with $\hat{x}(t)=\left[\begin{array}{llll}\hat{x}_{1}^{T}(t) & \hat{x}_{2}^{T}(t) \ldots \hat{x}_{M}^{T}(t)\end{array}\right]^{T}$, where

$$
\hat{x}_{i}(t)=\left\{\begin{array}{l}
x_{i n i t}^{i}, \forall t \in\left[0, a_{0}^{i}\right) \\
x_{i}\left(t-\tau_{i}(t)\right), \forall t \in\left[a_{k}^{i}, a_{k+1}^{i}\right), k \in \mathbb{N}
\end{array}\right.
$$

for all $i \in\{1,2, \ldots, M\}$, and

$$
\tau_{i}(t)=t-s_{k}^{i}, \forall t \in\left[a_{k}^{i}, a_{k+1}^{i}\right), k \in \mathbb{N} .
$$

Based on the $\mathcal{L}_{2}$-stability of the feedback interconnection $\mathbf{G}-\boldsymbol{\Delta}$, we have proved above that the solution $x(t)$ of the homogeneous system (57)-(59) belongs to $\mathcal{L}_{2 e}^{n}$. In order to prove exponential stability of the equilibrium point $x=0$, we shall invoke the Bohl-Perron Principle [9]. In accordance with the Bohl-Perron Principle, we shall prove that in the presence of an additional disturbance belonging to $\mathcal{L}_{2}^{n}$, the solution of the decentralized system (57)-(59) belongs to $\mathcal{L}_{2}^{n}$. To this end, consider the system

$$
y_{\star}(t)=\dot{x}_{\star}(t)=A x_{\star}(t)+B K \hat{x}_{\star}(t)+w_{\star}(t), x_{\star}(0)=x_{0},
$$

with $x_{\star}(t)=\left[\begin{array}{llll}x_{1_{\star}}^{T}(t) & x_{2_{\star}}^{T}(t) \ldots x_{M_{\star}}^{T}(t)\end{array}\right]^{T}$ and $\hat{x}_{\star}(t)=$ $\left[\begin{array}{llll}\hat{x}_{1_{\star}}^{T}(t) & \hat{x}_{2_{\star}}^{T}(t) & \ldots & \hat{x}_{M_{\star}}^{T}(t)\end{array}\right]^{T}$, where

$$
\hat{x}_{i_{\star}}(t)=\left\{\begin{array}{l}
x_{i n i t}^{i}, \forall t \in\left[0, a_{0}^{i}\right), \\
x_{i_{\star}}\left(t-\tau_{i}(t)\right), \forall t \in\left[a_{k}^{i}, a_{k+1}^{i}\right), k \in \mathbb{N},
\end{array}\right.
$$

for all $i \in\{1,2, \ldots, M\}$, and the disturbance $w_{\star} \in \mathcal{L}_{2}^{n}$. In a similar manner as given in the proof of Theorem 2 , the system $(60),(61)$ can be shown to be equivalent to the feedback interconnection given by

$$
\begin{aligned}
& y_{z_{\star}}=\mathbf{G} u_{z_{\star}}, u_{z_{\star}}=g_{\star}+e_{\star}, \\
& e_{\star}=\Delta y_{\star}, y_{\star}=y_{z_{\star}}+\left(f_{\star}+w_{\star}\right)
\end{aligned}
$$

where $g_{\star}$ is given in a similar manner as shown in $(31)$, i.e., $g_{i_{\star}}(t)=\left\{\begin{array}{l}x_{i n i t}^{i}-\mu_{i_{\star}}(t), \forall t \in\left[0, a_{0}^{i}\right), \\ 0, \forall t \geq a_{0}^{i},\end{array} \quad\right.$ for all $i \in\{1,2, \ldots, M\}$, with $\mu_{i_{\star}}(t)$ defined by a duplicate system with dynamics similar to that of (60), for all $t \in\left[0, a_{0}^{\star}\right)$, and

$$
\begin{aligned}
f_{\star}(t)= & \tilde{A}_{c l} e^{\hat{A} t} x_{0}+\tilde{A}_{c l} \int_{0}^{t} e^{\tilde{A}_{c l}(t-\tau)} \tilde{A} e^{\hat{A} \tau} x_{0} d \tau \\
& +w_{\star}(t)+\tilde{A}_{c l} \int_{0}^{t} e^{\tilde{A}_{c l}(t-\tau)} w_{\star}(\tau) d \tau, \\
= & f(t)+w_{\star}(t)+\tilde{A}_{c l} \int_{0}^{t} e^{\tilde{A}_{c l}(t-\tau)} w_{\star}(\tau) d \tau .
\end{aligned}
$$

where $f(t) \in \mathcal{L}_{2 e}^{n}$ is given by (54), and the disturbance $w_{\star} \in \mathcal{L}_{2}^{n}$. Note that $f(t)$ also belongs to $\mathcal{L}_{2}^{n}$. The term 
$\int_{0}^{t} e^{\tilde{A}_{c l}(t-\tau)} w_{\star}(\tau) d \tau$ is the response of a stable LTI system to the input $w_{\star} \in \mathcal{L}_{2}^{n}$, which belongs to $\mathcal{L}_{2}^{n}$. Consequently, we have that $f_{\star} \in \mathcal{L}_{2}^{n}$. Additionally, the solution of the non-homogeneous decentralized system (60), (61) will be given by

$$
\begin{aligned}
x_{\star}(t)= & z_{\star}(t)+\int_{0}^{t} e^{\tilde{A}_{c l}(t-\tau)} \tilde{A} e^{\hat{A} \tau} x_{0} d \tau \\
& +\int_{0}^{t} e^{\tilde{A}_{c l}(t-\tau)} w_{\star}(\tau) d \tau+e^{\hat{A} t} x_{0},
\end{aligned}
$$

with $z_{\star}$ given by a system similar to (48), with the variables $z_{\star}, y_{z_{\star}}$ and $u_{z_{\star}}$ instead of $z, y$ and $u_{z}$, respectively. The aforementioned equivalence between feedback interconnection (62) and system (60), (61) can be easily verified by replacing $w_{i}(t)$ in $(32)$ with $w_{i}(t)+w_{i_{\star}}(t)$, and following the proof of Theorem 2 . Therefore, the $i^{t h}$ closed-loop in the decentralized setting (with the variable $x_{i_{\star}}$ ) can be remodelled as the feedback interconnection given in Figure 6 , but with the disturbance $w_{i}+w_{i_{\star}}$ on the operator $\mathbf{G}_{i}$. Consequently, the decentralized system can be remodelled in the form shown in Figure 7, but with the disturbance $\phi+w_{\star}$ on the operator $\hat{\mathbf{G}}$. Finally, by considering a system similar to (48), with the variables $z_{\star}, y_{z_{\star}}$ and $u_{z_{\star}}$ instead of $z, y$ and $u_{z}$, respectively, the equivalence between $x_{\star}$ and $z_{\star}$ given by (64), can be proved.

Since $g_{\star} \in \mathcal{L}_{2}^{n}$ and the feedback-interconnection $\mathbf{G}-\boldsymbol{\Delta}$ is $\mathcal{L}_{2}$-stable, we have that $y_{z_{\star}}, u_{z_{\star}} \in \mathcal{L}_{2}^{n}$. Therefore, we have proved that $z_{\star} \in \mathcal{L}_{2}^{n}$ and consequently, from (64), we have $x_{\star} \in \mathcal{L}_{2}^{n}$. Now, since the solution of the non-homogeneous decentralized system $(60),(61)$, i.e., $x_{\star} \in \mathcal{L}_{2}^{n}$, by virtue of Bohl-Perron Principle [9], we can conclude that the equilibrium solution $x=0$ of the homogeneous decentralized system (57), (58), is globally exponentially stable.

Remark: In Theorem 3, the condition that both $\tilde{A}_{c l}$ and $\hat{A}$ need to be Hurwitz, imposes an easy-to-satisfy constraint that in the absence of sampling and delay, the decentralized system (13)-(16) is asymptotically stable.

Theorem 3 shows that in order to prove that the decentralized system (13)-(16) is exponentially stable, it is enough to prove that the feedback-interconnection $\mathbf{G}-\boldsymbol{\Delta}$ given by $(25)$, is $\mathcal{L}_{2}$-stable. In the following section, we will provide tractable numerical stability criteria that guarantees $\mathcal{L}_{2}$-stability of the interconnection $\mathbf{G}-\boldsymbol{\Delta}$, by characterizing the properties of the operator $\boldsymbol{\Delta}$ given in (23) using an IQC. As such, these conditions also guarantee exponential stability of the decentralized system (13)-(16).

\subsection{IQC Characterization of Asynchrony Effect}

In this section, we study the properties of the operator $\boldsymbol{\Delta}$ in (23), with $\Delta_{i}$ defined analogous to (9), and characterize its gain properties using an IQC. The following lemma extends the result given in [15], to include an arbitrary number of sensors and actuators. We provide the proof for the sake of completeness.

Lemma 4 Consider $R=\operatorname{diag}\left(R_{1}, R_{2}, \ldots, R_{M}\right)$, with $R_{i} \in \mathbb{R}^{n_{i} \times n_{i}}, R_{i}=R_{i}^{T}>0$, for all $i \in\{1,2, \ldots, M\}$. Then, the operator $\boldsymbol{\Delta}$ defined by (23), with $\Delta_{i}$ defined analogous to (9), satisfies the IQC given by

$$
\int_{0}^{\infty}\left[\begin{array}{l}
y(t) \\
e(t)
\end{array}\right]^{T}\left[\begin{array}{cc}
S & 0 \\
0 & -R
\end{array}\right]\left[\begin{array}{l}
y(t) \\
e(t)
\end{array}\right] d t \geq 0
$$

where $e=\Delta y$ with $y(t)$ given by (53), and $S=$ $\operatorname{diag}\left(\gamma_{1}^{2} R_{1}, \gamma_{2}^{2} R_{2}, \ldots, \gamma_{M}^{2} R_{M}\right)$, with $\gamma_{i}=\bar{h}_{i}+\bar{\eta}_{i}$, for all $i=\{1,2, \ldots, M\}$.

Proof The proof is given in Appendix B.

The IQC characterizing the properties of operator $\boldsymbol{\Delta}$ can now be used to establish $\mathcal{L}_{2}$-stability of the feedback interconnection $\mathbf{G}-\boldsymbol{\Delta}$ defined by (25), as given in the following theorem. Consequently, as a result of Theorem 3 , the exponential stability of the decentralized system (13)-(16) is then also guaranteed.

Theorem 5 Consider the decentralized system defined by (13)-(16), and the transfer function

$$
\mathbf{G}(s)=\tilde{A}_{c l}\left(s I-\tilde{A}_{c l}\right)^{-1} B_{c l}+B_{c l}
$$

where $B_{c l}$ is given by (21). Suppose that $\tilde{A}_{c l}, \hat{A}$ given by (20), (29), respectively, are Hurwitz. If there exists $\epsilon>0$ such that

$$
\left[\begin{array}{c}
\mathbf{G}(j \omega) \\
I
\end{array}\right]^{T} \Pi\left[\begin{array}{c}
\mathbf{G}(j \omega) \\
I
\end{array}\right] \leq-\epsilon I
$$

is satisfied for all $\omega \in \mathbb{R}$, and

$$
\Pi=\left[\begin{array}{cc}
S & 0 \\
0 & -R
\end{array}\right],
$$

with $R=\operatorname{diag}\left(R_{1}, R_{2}, \ldots, R_{M}\right), R_{i}=R_{i}^{T}>0$, and $S=$ $\operatorname{diag}\left(\gamma_{1}^{2} R_{1}, \gamma_{2}^{2} R_{2}, \ldots, \gamma_{M}^{2} R_{M}\right)$ with $\gamma_{i}=\bar{h}_{i}+\bar{\eta}_{i}$ for all $i \epsilon$ $\{1,2, \ldots, M\}$, then, the origin is a globally exponentially stable solution of the decentralized system (13)-(16).

Proof From Lemma 4, we have that the operator $\boldsymbol{\Delta}$ satisfies the IQC defined by $\Pi$. Consequently, by invoking the standard IQC Theorem [18], we have that the 
mapping $\left[\begin{array}{l}f \\ g\end{array}\right] \mapsto\left[\begin{array}{c}y \\ u_{z}\end{array}\right]$ defined by the feedback interconnection $\mathbf{G}-\boldsymbol{\Delta}$ in $(25)$ is $\mathcal{L}_{2}$-stable if the condition (67) is satisfied. Then, as a direct application of Theorem 3, since the feedback interconnection of $\mathbf{G}$ and $(\boldsymbol{\Delta})$ is $\mathcal{L}_{2^{-}}$ stable, the decentralized system (13)-(16) is exponentially stable.

Remark: By applying the Kalman-Yakubovich-Popov Lemma, we can infer that the frequency-domain criterion given by (67) is equivalent to the existence of matrices $P=P^{T}>0$ and $R_{i}=R_{i}^{T}>0$, such that the Linear Matrix Inequality (LMI)

$$
\left[\begin{array}{cc}
\tilde{A}_{c l}^{T} P+P \tilde{A}_{c l} & P B_{c l} \\
B_{c l}^{T} P & 0
\end{array}\right]+\left[\begin{array}{cc}
\tilde{A}_{c l} & B_{c l} \\
0 & I
\end{array}\right]^{T} \Pi\left[\begin{array}{cc}
\tilde{A}_{c l} & B_{c l} \\
0 & I
\end{array}\right]<0
$$

where $\Pi$ is given by (68), and where $\tilde{A}_{c l}, B_{c l}$ are given by (20), (21), respectively, is satisfied. This condition can easily be checked using existing LMI solvers. For a set of asynchronous sampling intervals and delays, the condition allows to validate system stability. This is shown via a numerical example in Section 5 . For a single system setting, when $\bar{h}_{1}=0$, the condition (69) recovers the result given in [16]. Similarly, when $\bar{\eta}_{1}=0$, we recover the condition given in [19]. Richer IQC characterizations that account for passivity properties of operators similar to $\Delta$, in addition to the bounded gain properties, can be found in $[3,4,6,14]$. These characterizations could be useful in future work to derive less conservative stability criteria.

\section{Small Delay Case: Separation of $\Delta$ operator}

The large-delay case considered in Section 3 delineates scenarios commonly arising in data transmission over shared networks, where delays can be considerably longer than the sampling intervals. However, in a relevant subset of practical scenarios, the delays can be guaranteed to be smaller than the sampling interval. Such scenarios and its impact on the stability of systems has previously been illustrated in [22]. It was shown that for an exemplary LTI system, a specific sequence of alternating time delays, with the delay being less than the sampling interval, induced instability in the system. In such scenarios, the result presented in Theorem 5, i.e., for the large-delay case, can be applied. However, since asynchrony effects are analysed in between actuation instants, using a global operator, which does not distinguish between asynchrony induced by sampling, and delay, the obtained results are conservative when adapted to the small-delay case. In this section, we show how in the small-delay case, a distinction can be made between asynchrony induced by sampling and asynchrony induced by delay, by considering two operators.
This distinction also aids in obtaining less conservative results, when compared to the results obtained in the large-delay case, adapted to the small-delay case. We proceed to provide a mathematical description of the decentralized setting (13)-(16), in the small-delay case.

\subsection{System description}

In this section, we recall the decentralized sampled-data system (13)-(16). In the small-delay case, it holds for the $i^{t h}$-loop in the decentralized setting that the $k^{\text {th }}$ actuation instant occurs before the $(k+1)^{t h}$ sampling instant, i.e.,

$$
\eta_{k}^{i} \leq h_{k}^{i}, \forall k \in \mathbb{N}, i \in\{1,2, \ldots, M\} .
$$

Exploiting this more stringent requirement on the network, we proceed to provide a criteria that is less conservative in comparison to the more generic criteria given in Theorem 5, when applied to this small-delay case. As a stepping stone, we consider two operators to characterize the effects of sampling and delay separately, by adapting a similar formulation we have provided in [24]. The error due to sampling is given by $e_{s}(t)=\left[\begin{array}{llll}e_{1}^{s T}(t) & e_{2}^{s T}(t) & \ldots & e_{M}^{s} T(t)\end{array}\right]^{T}$, where

$e_{i}^{s}(t)=\left(\Delta_{i}^{s} y_{i}\right)(t)=\left\{\begin{array}{l}0, \forall t \in\left[0, a_{0}^{i}\right), \\ -\int_{s_{0}^{i}}^{t} y_{i}(\theta) d \theta, \forall t \in\left[a_{0}^{i}, s_{1}^{i}\right), \\ -\int_{s_{k}^{i}}^{t} y_{i}(\theta) d \theta, \forall t \in\left[s_{k}^{i}, s_{k+1}^{i}\right), k \in \mathbb{N}^{\star}\end{array}\right.$

for all $i \in\{1,2, \ldots, M\}$. In a similar manner, the error induced on a sampled signal due to delay, is given by $e_{d}(t)=\left[\begin{array}{llll}e_{1}^{d^{T}}(t) & e_{2}^{d^{T}}(t) & \ldots & e_{M}^{d^{T}}(t)\end{array}\right]^{T}$, where for all $i \epsilon$ $\{1,2, \ldots, M\}$,

$$
e_{i}^{d}(t)=\left(\Delta_{i}^{d} y_{i}\right)(t)=\left\{\begin{array}{l}
0, \forall t \in\left[0, s_{1}^{i}\right), \\
-\int_{s_{k-1}^{i}}^{s_{k}^{i}} y_{i}(\theta) d \theta, \forall t \in\left[s_{k}^{i}, a_{k}^{i}\right), k \in \mathbb{N}^{\star} \\
0, \forall t \in\left[a_{k}^{i}, s_{k+1}^{i}\right), k \in \mathbb{N}^{\star} .
\end{array}\right.
$$

\subsection{Operator decomposition}

In the following lemma, for the decentralized system (13)-(16) under the constraint (70), we demonstrate the equivalence between the error captured using a single operator, i.e., (38), and the error captured using two separate operators, i.e., (71) and (72).

Lemma 6 Consider the operator $\boldsymbol{\Delta}^{\mathbf{s}}$ given by

$$
\boldsymbol{\Delta}^{\mathbf{s}} y=\left(\begin{array}{llll}
\Delta_{1}^{s} y_{1} & \Delta_{2}^{s} y_{2} & \ldots & \Delta_{M}^{s} y_{M}
\end{array}\right)^{T}
$$

where, for all $i \in\{1,2, \ldots, M\}$, the operator $\Delta_{i}^{s}$ is defined 
by (71). Consider the operator $\boldsymbol{\Delta}^{\mathbf{d}}$ given by

$$
\boldsymbol{\Delta}^{\mathbf{d}} y=\left(\begin{array}{llll}
\Delta_{1}^{d} y_{1} & \Delta_{2}^{d} y_{2} & \ldots & \Delta_{M}^{d} y_{M}
\end{array}\right)^{T},
$$

where, for all $i \in\{1,2, \ldots, M\}$, the operator $\Delta_{i}^{d}$ is defined by (72). Then, for the decentralized sampled-data system (13)-(16) under constraint (70),

$$
(\Delta y)(t)=\left(\Delta^{\mathbf{s}} y\right)(t)+\left(\Delta^{\mathbf{d}} y\right)(t), \forall t \geq 0
$$

where the operator $\boldsymbol{\Delta}=\operatorname{diag}\left(\Delta_{1}, \Delta_{2}, \ldots, \Delta_{M}\right)$, so that for all $i \in\{1,2, \ldots, M\}, \Delta_{i}$ is defined by (38).

Proof Based on the structure of the operators $\Delta, \Delta^{\mathbf{s}}$, and $\boldsymbol{\Delta}^{\mathbf{d}}$, in order to prove $(75)$, it is sufficient to show that

$$
\left(\Delta_{i} y_{i}\right)(t)=\left(\Delta_{i}^{s} y_{i}\right)(t)+\left(\Delta_{i}^{d} y_{i}\right)(t), \forall t \geq 0
$$

where $\Delta_{i}$ is defined by $(38)$.

For all $t \in\left[0, a_{0}^{i}\right)$ : From (71), (72), and (38), we have $\left(\Delta_{i}^{s} y_{i}\right)(t)=\left(\Delta_{i}^{d} y_{i}\right)(t)=\left(\Delta_{i} y_{i}\right)(t)=0$, implying $\left(\Delta_{i}^{s} y_{i}\right)(t)+\left(\Delta_{i}^{d} y_{i}\right)(t)=\left(\Delta_{i} y_{i}\right)(t), \forall t \in\left[0, a_{0}^{i}\right)$.

For all $t \in\left[a_{0}^{i}, s_{1}^{i}\right)$ : From the definition of $\Delta_{i}$ in (38), we have

$$
\left(\Delta_{i} y_{i}\right)(t)=-\int_{s_{0}^{i}}^{t} y_{i}(\theta) d \theta, \forall t \in\left[a_{0}^{i}, a_{1}^{i}\right) \supset\left[a_{0}^{i}, s_{1}^{i}\right)
$$

From (72), since $\left(\Delta_{i}^{d} y_{i}\right)(t)=0$, for all $t \in\left[a_{0}^{i}, s_{1}^{i}\right)$, using the definition of $\Delta_{i}^{s}$ in (71), (77) can be expressed as

$$
\left(\Delta_{i} y_{i}\right)(t)=\left(\Delta_{i}^{s} y_{i}\right)(t)+\left(\Delta_{i}^{d} y_{i}\right)(t), \forall t \in\left[a_{0}^{i}, s_{1}^{i}\right)
$$

For all $t \in\left[s_{k}^{i}, a_{k}^{i}\right), k \in \mathbb{N}^{\star}:$ From (71) and (72), we have

$$
\begin{aligned}
& \left(\Delta_{i}^{s} y_{i}\right)(t)+\left(\Delta_{i}^{d} y_{i}\right)(t) \\
& =-\int_{s_{k}^{i}}^{t} y_{i}(\theta) d \theta-\int_{s_{k-1}^{i}}^{s_{k}^{i}} y_{i}(\theta) d \theta, \forall t \in\left[s_{k}^{i}, a_{k}^{i}\right), k \in \mathbb{N}^{\star} \\
& =-\int_{s_{k-1}^{i}}^{t} y_{i}(\theta) d \theta, \forall t \in\left[s_{k}^{i}, a_{k}^{i}\right), k \in \mathbb{N}^{\star}
\end{aligned}
$$

From (38), under the condition (70), we have

$$
\begin{aligned}
& \left(\Delta_{i} y_{i}\right)(t)=-\int_{s_{k}^{i}}^{t} y_{i}(\theta) d \theta \\
& \forall t \in\left[a_{k}^{i}, a_{k+1}^{i}\right) \supset\left[s_{k+1}^{i}, a_{k+1}^{i}\right), k \in \mathbb{N}, \\
& =-\int_{s_{p-1}^{i}}^{t} y_{i}(\theta) d \theta, \forall t \in\left[a_{p-1}^{i}, a_{p}^{i}\right) \supset\left[s_{p}^{i}, a_{p}^{i}\right), p \in \mathbb{N}^{\star} .
\end{aligned}
$$

Therefore, (79) gives

$$
\left(\Delta_{i}^{s} y_{i}\right)(t)+\left(\Delta_{i}^{d} y_{i}\right)(t)=\left(\Delta_{i} y_{i}\right)(t), \forall t \in\left[s_{k}^{i}, a_{k}^{i}\right), k \in \mathbb{N}^{\star}
$$

For all $t \in\left[a_{k}^{i}, s_{k+1}^{i}\right), k \in \mathbb{N}^{\star}:$ Using (71) and (72), we

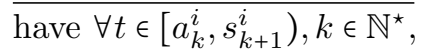

$$
\left(\Delta_{i}^{s} y_{i}\right)(t)+\left(\Delta_{i}^{d} y_{i}\right)(t)=-\int_{s_{k}^{i}}^{t} y(\theta) d \theta
$$

Now, from the definition of $\Delta_{i}$ in (38), under constraint (70), we can state

$$
\begin{aligned}
& \left(\Delta_{i} y_{i}\right)(t)=-\int_{s_{k+1}^{i}}^{t} y_{i}(\theta) d \theta \\
& \forall t \in\left[a_{k+1}^{i}, a_{k+2}^{i}\right) \supset\left[a_{k+1}^{i}, s_{k+2}^{i}\right), k \in \mathbb{N}, \\
& =-\int_{s_{p}^{i}}^{t} y_{i}(\theta) d \theta, \forall t \in\left[a_{p}^{i}, a_{p+1}^{i}\right) \supset\left[a_{p}^{i}, s_{p+1}^{i}\right), p \in \mathbb{N}^{\star} .
\end{aligned}
$$

Therefore (83) and (82) gives

$\left(\Delta_{i}^{s} y_{i}\right)(t)+\left(\Delta_{i}^{d} y_{i}\right)(t)=\left(\Delta_{i} y_{i}\right)(t), \forall t \in\left[a_{k}^{i}, s_{k+1}^{i}\right), k \in \mathbb{N}^{\star}$.

Hence, from (76), (78), (81) and (84), we have $\left(\Delta_{i}^{s} y_{i}\right)(t)+\left(\Delta_{i}^{d} y_{i}\right)(t)=\left(\Delta_{i} y_{i}\right)(t), \forall t \geq 0$.

In a similar fashion as demonstrated in the large-delay case, we proceed to characterize the properties of the operators $\boldsymbol{\Delta}^{\mathbf{s}}$ and $\boldsymbol{\Delta}^{\mathbf{d}}$, given by (73) and (74), respectively, using IQCs. By doing so, we can provide IQC conditions that guarantee $\mathcal{L}_{2}$-stability of the feedback interconnection $\mathbf{G}-\boldsymbol{\Delta}$, with $\boldsymbol{\Delta}$ satisfying the decomposition (75).

\subsection{IQC Characterization}

In this section, we characterize the properties of the operators $\boldsymbol{\Delta}^{\mathbf{s}}$ and $\boldsymbol{\Delta}^{\mathbf{d}}$ using IQCs. The following lemma provides IQC conditions on the operator $\boldsymbol{\Delta}^{\mathbf{s}}$, that characterizes the effects of asynchrony induced by sampling and hold. The result given in this lemma is an extension of the results provided in $[17,19]$, wherein a single-loop LTI system with aperiodic sampling was considered.

Lemma 7 Consider $R_{s}=\operatorname{diag}\left(R_{1}^{s}, R_{2}^{s}, \ldots, R_{M}^{s}\right)$, with $R_{i}^{s} \in \mathbb{R}^{n_{i} \times n_{i}}, R_{i}^{s}=\left(R_{i}^{s}\right)^{T}>0$, for all $i \in\{1,2, \ldots, M\}$. The operator $\boldsymbol{\Delta}^{\mathbf{s}}$ defined by (73) satisfies the Integral Quadratic Constraint (IQC) given by

$$
\int_{0}^{\infty}\left[\begin{array}{c}
y(t) \\
e_{s}(t)
\end{array}\right]^{T}\left[\begin{array}{cc}
S_{s} & 0 \\
0 & -R_{s}
\end{array}\right]\left[\begin{array}{c}
y(t) \\
e_{s}(t)
\end{array}\right] d t \geq 0
$$

where $y$ is given by $(53), e_{s}=\Delta^{\mathbf{s}} y$, and

$$
S_{s}=\operatorname{diag}\left(\left(\gamma_{1}^{s}\right)^{2} R_{1}^{s},\left(\gamma_{2}^{s}\right)^{2} R_{2}^{s}, \ldots,\left(\gamma_{M}^{s}\right)^{2} R_{M}^{s}\right),
$$

with $\gamma_{i}^{s}=\frac{2 \bar{h}_{i}}{\pi}$, for all $i=\{1,2, \ldots, M\}$

Proof The proof is given in Appendix C. 
In a similar manner as shown in Lemma 7 , in the following lemma, we characterize the properties of the operator $\boldsymbol{\Delta}^{\mathbf{d}}$, that characterizes the effects of asynchrony induced by delay, using an IQC.

Lemma 8 Consider $R_{d}=\operatorname{diag}\left(R_{1}^{d}, R_{2}^{d}, \ldots, R_{M}^{d}\right)$, with $R_{i}^{d} \in \mathbb{R}^{n_{i} \times n_{i}}, R_{i}^{d}=\left(R_{i}^{d}\right)^{T}>0$, for all $i \in\{1,2, \ldots, M\}$. The operator (74) satisfies the Integral Quadratic Constraint (IQC) given by

$$
\int_{0}^{\infty}\left[\begin{array}{c}
y(t) \\
e_{d}(t)
\end{array}\right]^{T}\left[\begin{array}{cc}
S_{d} & 0 \\
0 & -R_{d}
\end{array}\right]\left[\begin{array}{c}
y(t) \\
e_{d}(t)
\end{array}\right] d t \geq 0
$$

where $y(t)$ is given by (53), $e_{d}=\boldsymbol{\Delta}^{\mathbf{d}} y$, and

$$
S_{d}=\operatorname{diag}\left(\left(\gamma_{1}^{d}\right)^{2} R_{1}^{d},\left(\gamma_{2}^{d}\right)^{2} R_{2}^{d}, \ldots,\left(\gamma_{M}^{d}\right)^{2} R_{M}^{d}\right),
$$

with $\gamma_{i}^{d}=\sqrt{\bar{h}_{i} \bar{\eta}_{i}}$, for all $i=\{1,2, \ldots, M\}$.

Proof Consider the delay-induced error given by (72). We have,

$$
\begin{aligned}
\int_{s_{1}^{i}}^{\infty} e_{i}^{d}(t)^{T} R_{i}^{d} e_{i}^{d}(t) d t & =\sum_{k=1}^{\infty} \int_{s_{k}^{i}}^{s_{k+1}^{i}} e_{i}^{d}(t)^{T} R_{i}^{d} e_{i}^{d}(t) d t \\
& =\sum_{k=1}^{\infty} \int_{s_{k}^{i}}^{a_{k}^{i}} e_{i}^{d}(t)^{T} R_{i}^{d} e_{i}^{d}(t) d t
\end{aligned}
$$

since $e_{i}^{d}(t)=0$ for all $t \in\left[a_{k}^{i}, s_{k+1}^{i}\right), k \in \mathbb{N}^{\star}$. Since $e_{i}^{d}(t):=-\int_{s_{k-1}^{i}}^{s_{k}^{i}} y_{i}(\theta) d \theta, \forall t \in\left[s_{k}^{i}, a_{k}^{i}\right), k \in \mathbb{N}^{\star}$, by employing Jensen's inequality, we obtain

$$
e_{i}^{d}(t)^{T} R_{i}^{d} e_{i}^{d}(t) \leq \bar{h}_{i} \int_{s_{k-1}^{i}}^{s_{k}^{i}} y_{i}(\theta)^{T} R_{i}^{d} y_{i}(\theta) d \theta, k \in \mathbb{N}^{\star}
$$

Using the bound (90), we have from (89) that

$$
\begin{aligned}
\int_{s_{1}^{i}}^{\infty} e_{i}^{d}( & t)^{T} R_{i}^{d} e_{i}^{d}(t) d t \\
& \leq \bar{h}_{i} \sum_{k=1}^{\infty} \int_{s_{k}^{i}}^{a_{k}^{i}}\left(\int_{s_{k-1}^{i}}^{s_{i}^{i}} y_{i}(\theta)^{T} R_{i}^{d} y_{i}(\theta) d \theta\right) d t \\
& =\bar{h}_{i} \bar{\eta}_{i} \sum_{k=1}^{\infty} \int_{s_{k-1}^{i}}^{s_{i}^{i}} y_{i}(\theta)^{T} R_{i}^{d} y_{i}(\theta) d \theta \\
& \leq \bar{h}_{i} \bar{\eta}_{i} \int_{0}^{\infty} y_{i}(t)^{T} R_{i}^{d} y_{i}(t) d t .
\end{aligned}
$$

Since $e_{i}^{d}(t)=0$ for all $t \leq s_{1}^{i}$, we have $\int_{0}^{\infty} e_{i}^{d}(t)^{T} R_{i}^{d} e_{i}^{d}(t) d t$ $=\int_{s_{1}^{i}}^{\infty} e_{i}^{d}(t)^{T} R_{i}^{d} e_{i}^{d}(t) d t \leq \bar{h}_{i} \bar{\eta}_{i} \int_{0}^{\infty} y_{i}(t)^{T} R_{i}^{d} y_{i}(t) d t$. Consequently, for all $i=\{1,2, \ldots, M\}$, we have

$$
\int_{0}^{\infty}\left[\begin{array}{l}
y_{i}(t) \\
e_{i}^{d}(t)
\end{array}\right]^{T}\left[\begin{array}{cc}
\gamma_{i}^{d^{2}} R_{i}^{d} & 0 \\
0 & -R_{i}^{d}
\end{array}\right]\left[\begin{array}{c}
y(t) \\
e_{i}^{d}(t)
\end{array}\right] d t \geq 0,
$$

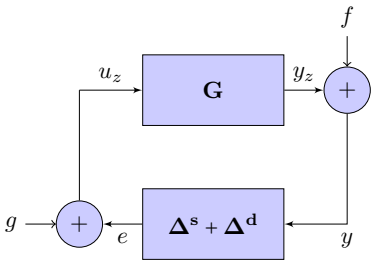

Fig. 8. The feedback interconnection of $\mathbf{G}$ and $\boldsymbol{\Delta}^{\mathbf{s}}+\boldsymbol{\Delta}^{\mathrm{d}}$, representing the decentralized system (13)-(16).

where $\gamma_{i}^{d}=\sqrt{\bar{h}_{i} \bar{\eta}_{i}}$. Considering the integral quadratic constraint (92) for all $i \in\{1,2, \ldots, M\}$, i.e., for the operator $\boldsymbol{\Delta}^{\mathbf{s}}$, we have

$$
\int_{0}^{\infty}\left[\begin{array}{c}
y(t) \\
e_{d}(t)
\end{array}\right]^{T}\left[\begin{array}{cc}
S_{d} & 0 \\
0 & -R_{d}
\end{array}\right]\left[\begin{array}{c}
y(t) \\
e_{d}(t)
\end{array}\right] d t \geq 0
$$

where

$$
\begin{aligned}
e_{d}(t) & =\left[e_{1}^{d^{T}}(t), e_{2}^{d^{T}}(t), \ldots, e_{M}^{d}(t)\right]^{T}, \\
y(t) & =\left[y_{1}^{T}(t), y_{2}^{T}(t), \ldots, y_{M}^{T}(t)\right]^{T}, \\
S_{d} & =\operatorname{diag}\left(\left(\gamma_{1}^{d}\right)^{2} R_{1}^{d},\left(\gamma_{2}^{d}\right)^{2} R_{2}^{d}, \ldots,\left(\gamma_{M}^{d}\right)^{2} R_{M}^{d}\right) .
\end{aligned}
$$

We have now characterized the properties of operators $\boldsymbol{\Delta}^{\mathbf{s}}$ and $\boldsymbol{\Delta}^{\mathbf{d}}$ using IQCs. In a similar manner as shown in the large-delay case, this will be used to provide tractable numerical conditions that guarantee $\mathcal{L}_{2}$-stability of the feedback interconnection $\mathbf{G}-\boldsymbol{\Delta}$, where $\boldsymbol{\Delta}$ satisfies the decomposition (75), and ultimately global exponential stability of the decentralized system (13)-(16).

\subsection{Exponential Stability Criterion}

In this section, based on the IQCs characterizing operators $\boldsymbol{\Delta}^{\mathbf{s}}$ and $\boldsymbol{\Delta}^{\mathrm{d}}$, we establish the $\mathcal{L}_{2}$-stability of the feedback interconnection $\mathbf{G}-\boldsymbol{\Delta}$. By doing so, in conjunction with Theorem 3 , we are able to guarantee the exponential stability of decentralized system (13)-(16).

Theorem 9 Consider the decentralized system defined by (13)-(16), and the transfer function

$$
\mathbf{G}(s)=\tilde{A}_{c l}\left(s I-\tilde{A}_{c l}\right)^{-1} B_{c l}+B_{c l} .
$$

where $B_{c l}$ is given by (21). Suppose that $\tilde{A}_{c l}, \hat{A}$ given by (20), (29), respectively, are Hurwitz. If there exists $\epsilon>0$ such that

$$
\left[\begin{array}{c}
\mathbf{G}(j \omega) \\
I
\end{array}\right]^{T} \tilde{\Pi}\left[\begin{array}{c}
\mathbf{G}(j \omega) \\
I
\end{array}\right] \leq-\epsilon I,
$$


is satisfied for all $\omega \in \mathbb{R}$, and

$$
\tilde{\Pi}=\left[\begin{array}{ccc}
S_{s}+S_{d} & 0 & 0 \\
0 & -R_{s} & 0 \\
0 & 0 & -R_{d}
\end{array}\right],
$$

with $S_{s}, S_{d}$ given by (86), (88), respectively, $R_{s}=$ $\operatorname{diag}\left(R_{1}^{s}, R_{2}^{s}, \ldots, R_{M}^{s}\right), R_{d}=\operatorname{diag}\left(R_{1}^{d}, R_{2}^{d}, \ldots, R_{M}^{d}\right)$, so that $R_{i}^{s}=\left(R_{i}^{s}\right)^{T}>0$, and $R_{i}^{d}=\left(R_{i}^{d}\right)^{T}>0$ for all $i \in\{1,2, \ldots, M\}$, then, the decentralized system (13)(16) is globally exponentially stable.

Proof Based on Lemma 6, since $(\boldsymbol{\Delta} y)(t)=\left(\boldsymbol{\Delta}^{\mathbf{s}} y\right)(t)+$ $\left(\boldsymbol{\Delta}^{\mathbf{d}} y\right)(t), \forall t \geq 0$, we have that $e(t)=e_{s}(t)+e_{d}(t), \forall t \geq$ 0 , where $e_{s}(t)$ and $e_{d}(t)$ are given by (C.3) and (94), respectively. Consequently, using the feedback interconnection $\mathbf{G}-\boldsymbol{\Delta}$ given by Figure 5, we can represent the decentralized system (13)-(16) by the feedback interconnection given in Figure 8. Additionally, using Lemmas 7 and 8 , we have that $\boldsymbol{\Delta}^{\mathrm{s}}+\boldsymbol{\Delta}^{\mathrm{d}}$ satisfies the IQC given by

$$
\int_{0}^{\infty}\left[\begin{array}{c}
y(t) \\
e_{s}(t) \\
e_{d}(t)
\end{array}\right]^{T} \tilde{\Pi}\left[\begin{array}{c}
y(t) \\
e_{s}(t) \\
e_{d}(t)
\end{array}\right] d t \geq 0,
$$

where $\tilde{\Pi}$ is given by (97). Therefore by invoking the IQC Theorem [18], we can state that the feedbackinterconnection of the operators $\mathbf{G}$ and $\left(\boldsymbol{\Delta}^{\mathrm{s}}+\boldsymbol{\Delta}^{\mathrm{d}}\right)$ is $\mathcal{L}_{2}$-stable if the IQC condition (96) is satisfied. Then, as a direct application of Theorem 3, since the feedback interconnection of $\mathbf{G}$ and $\left(\boldsymbol{\Delta}^{\mathbf{s}}+\boldsymbol{\Delta}^{\mathbf{d}}\right)$ is $\mathcal{L}_{2}$-stable, the decentralized system (13)-(16) is exponentially stable.

Remark: By applying the Kalman-Yakubovich-Popov Lemma, we can infer that the frequency-domain criterion given by (96) is equivalent to the existence of $P=P^{T}>0, R_{i}^{s}=\left(R_{i}^{s}\right)^{T}>0, R_{i}^{d}=\left(R_{i}^{d}\right)^{T}>0$, such that the LMI given by

$$
\left[\begin{array}{cc}
\tilde{A}_{c l}^{T} P+P \tilde{A}_{c l} & P \bar{B} \\
\bar{B}^{T} P & 0
\end{array}\right]+\left[\begin{array}{cc}
\tilde{A}_{c l} & \bar{B} \\
0 & I
\end{array}\right]^{T} \tilde{\Pi}\left[\begin{array}{cc}
\tilde{A}_{c l} & \bar{B} \\
0 & I
\end{array}\right]<0
$$

where $\tilde{\Pi}$ is given by $(97)$, and $\bar{B}=\left[\begin{array}{ll}B_{c l} & B_{c l}\end{array}\right]$, is satisfied. The matrices $\tilde{A}_{c l}$ and $B_{c l}$ are given by (20) and (21), respectively.

\section{$5 \quad$ Numerical Example}

In this section, we consider again the motivating example given in the introduction, also studied in [24], and given

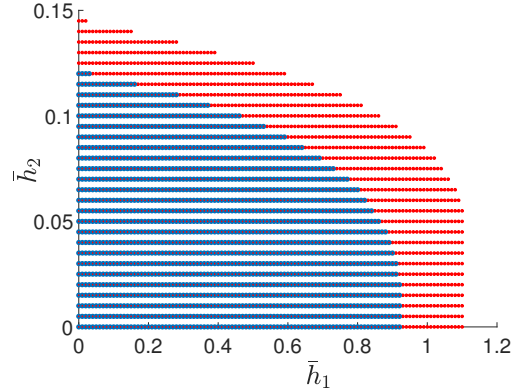

Fig. 9. Feasible values of $\bar{h}_{1}$ and $\bar{h}_{2}$, when $\bar{\eta}_{1}=\bar{\eta}_{2}=0.075$, for the large-delay case (in blue) and the small-delay case (in red). For the small-delay case, the bounds $\bar{h}_{1}<0.075$ and $\bar{h}_{2}<0.075$ are applicable only if the condition (70) is satisfied.

by the matrices

$$
A=\left[\begin{array}{cc}
-2 & -1 \\
2.8 & 4
\end{array}\right], B=\left[\begin{array}{ll}
1 & 0 \\
0 & 1
\end{array}\right], K=\left[\begin{array}{cc}
-1 & 0 \\
0 & -4.6
\end{array}\right] .
$$

One of the prime advantages of the results obtained in this paper, compared to the results in [24], is that the stability conditions introduced in this paper can be checked for any sampling and delay upper-bounds, for each sensor, individually. In this example, we can therefore check the system's stability for any quadruplet $\left(\bar{h}_{1}, \bar{h}_{2}, \bar{\eta}_{1}, \bar{\eta}_{2}\right)$. We will now illustrate as follows, how the results proposed in this paper aid in computing the feasibile values of individual sampling interval bounds, i.e., $\bar{h}_{1}$ and $\bar{h}_{2}$, separately, for fixed delays. In Figure 9, we show for instance, the stability domain obtained with fixed delay upper-bounds $\bar{\eta}_{1}=\bar{\eta}_{2}=0.075$.

The feasible values of $\bar{h}_{1}$ and $\bar{h}_{2}$ are computed for $\bar{\eta}_{1}=$ $\bar{\eta}_{2}=0.075$, in the large-delay case (in blue) and the small-delay case (in red), and are shown in Figure 9. It is evident from the figure that the criterion proposed for the small-delay case provides less conservative results, in comparison to the criterion introduced for the largedelay case, but adapted to the small-delay scenario. The advantage of the large-delay case, as mentioned previously, is that it allows for the delay $\eta_{i}^{k}, i \in\{1,2\}, k \in \mathbb{N}$, to be greater than the sampling interval $h_{i}^{k}, i \in\{1,2\}, k \in \mathbb{N}$, for any feasible point $\left(\bar{h}_{1}, \bar{h}_{2}\right)$ chosen in the blue feasibility region, as long as the sampling and actuation instants satisfy the large-delay constraint given in (15). On the contrary, for a feasible point $\left(\bar{h}_{1}, \bar{h}_{2}\right)$ in the red feasibility region, $h_{i}^{k}, i \in\{1,2\}, k \in \mathbb{N}$ and $\eta_{i}^{k}, i \in\{1,2\}, k \in \mathbb{N}$ need to satisfy the more restrictive small-delay constraint given by (70). A plot providing the feasible values of $\bar{h}_{1}$ and $\bar{h}_{2}$, can also be obtained for fixed delay bounds with $\bar{\eta}_{1} \neq \bar{\eta}_{2}$.

In order to illustrate that the stability criteria proposed in this paper are less conservative compared to the criteria provided in [24], we study the maximum bound on sampling interval, so that $\bar{h}_{1}=\bar{h}_{2}$, when delay bounds 
are set to zero, i.e., $\bar{\eta}_{1}=\bar{\eta}_{2}=0$. For the large-delay case, by virtue of Theorem 5 , by solving the LMI (69), we obtain $\bar{h}_{1}=\bar{h}_{2}=0.19$, in comparison to a bound of $0.18 \mathrm{ob}-$ tained in [24]. Similarly, for the small-delay case, applying Theorem 9, i.e., LMI (99), we obtain $\bar{h}_{1}=\bar{h}_{2}=0.31$, whereas a bound of 0.27 was obtained in [24].

The example shown in this section gives an insight into how the tools proposed in this paper can be used to decide the trade-off between sampling-interval bounds and delays, depending upon the system under consideration, and the constraints imposed by the networked communication channel. As a result, separate, effective sampling and actuation strategies can be employed on individual sensors and actuators, respectively.

\section{Conclusion}

In this paper, a novel, IQC based framework towards exponential stability analysis of state-space models of decentralized, sampled-data LTI control systems with asynchronous sensors and actuators, is provided. As a preliminary result, an approach is introduced to represent the state-space model of a single-loop LTI system with asynchronous sensors and actuators, as an interconnection between a continuous time system operator and an operator that captures the effects of asynchrony. Consequently, by scaling this preliminary result, the decentralized, sampled-data, asynchronous LTI state-space model under consideration, is reformulated as a feedback interconnection. By characterizing the properties of the operator that captures asynchrony effects, using an IQC, stability results on the feedbackinterconnection, which imply global exponential stability of the decentralized system, are provided. Two scenarios, namely the large-delay case and the smalldelay case, are considered. In the large-delay case, the effects of asynchrony induced by sampling and delay, are captured using a single operator. In contrast, these effects are captured using two separate operators in the small-delay case. This leads to less conservative results, in comparison to the result obtained in the large-delay case, when adapted to the small-delay case. The effectiveness of the proposed results have been illustrated using a numerical example. Future work considering richer IQC characterizations of the operator capturing sampling and asynchrony effects can be useful in deriving less conservative results.

\section{Acknowledgements}

This work was supported by project UCoCoS, funded by the European Union's EU Framework Programme for Research and Innovation, Horizon H2020, Grant Agreement No: 675080 .

\section{References}

[1] L. Bakule and J. Lunze. Decentralized Design of Feedback Control for Large-scale Systems. Kybernetika: Příloha. Academia, 1988.

[2] Lubomír Bakule. Decentralized control: An overview. Annual Reviews in Control, 32(1):87 98, 2008.

[3] M. Cantoni, M. A. Fabbro, and C. Kao. Stability of aperiodic sampled-data feedback for systems with inputs that update asynchronously. In 2018 IEEE Conference on Decision and Control (CDC), pages 7118-7123, Dec 2018.

[4] M. Cantoni, C. Y. Kao, and M. A. Fabbro. Integral quadratic constraints for asynchronous sampleand-hold links. IEEE Transactions on Automatic Control, pages 1-1, 2020.

[5] V. S. Dolk, D. P. Borgers, and W. P. M. H. Heemels. Output-based and decentralized dynamic eventtriggered control with guaranteed $\mathcal{L}_{p^{-}}$gain performance and zeno-freeness. IEEE Transactions on Automatic Control, 62(1):34-49, Jan 2017.

[6] Mark A. Fabbro, Michael Cantoni, and ChungYao Kao. Performance analysis of feedback loops with asynchronous sampling in the forward and return paths. IFAC-PapersOnLine, 49(22):256 - 261, 2016. 6th IFAC Workshop on Distributed Estimation and Control in Networked Systems NECSYS 2016.

[7] C. Fiter, T.-E. Korabi, L. Etienne, and L. Hetel. Stability of LTI Systems with Distributed Sensors and Aperiodic Sampling, pages 63-82. Springer International Publishing, Cham, 2018.

[8] Dror Freirich and Emilia Fridman. Decentralized networked control of systems with local networks: A time-delay approach. Automatica, 69:201 - 209, 2016.

[9] E. Fridman. Introduction to Time-Delay Systems: Analysis and Control. Systems \& Control: Foundations \& Applications. Springer International Publishing, 2014.

[10] Emilia Fridman and Uri Shaked. Input-output approach to stability and 12-gain analysis of systems with time-varying delays. Systems $\&$ Control Letters, 55(12):1041 - 1053, 2006.

[11] Hisaya Fujioka. Stability analysis of systems with aperiodic sample-and-hold devices. Automatica, 45(3):771 - 775, 2009.

[12] Laurentiu Hetel, Christophe Fiter, Hassan Omran, Alexandre Seuret, Emilia Fridman, Jean Pierre Richard, and Silviu Iulian Niculescu. Recent developments on the stability of systems with aperiodic sampling: An overview. Automatica, 76:309 - 335, 2017.

[13] Myungsoo Jun and Michael G. Safonov. Iqc robustness analysis for time-delay systems. International Journal of Robust and Nonlinear Control, 11(15):1455-1468, 2001.

[14] C. Kao and M. Cantoni. Robust performance anal- 
ysis of aperiodic sampled-data feedback control systems. In 2015 54th IEEE Conference on Decision and Control (CDC), pages 1421-1426, 2015.

[15] Chung-Yao Kao and Bo Lincoln. Simple stability criteria for systems with time-varying delays. $A u$ tomatica, 40(8):1429 - 1434, 2004.

[16] Chung-Yao Kao and Anders Rantzer. Stability analysis of systems with uncertain time-varying delays. Automatica, 43(6):959 - 970, 2007.

[17] Kun Liu, Vladimir Suplin, and Emilia Fridman. Stability of linear systems with general sawtooth delay. IMA Journal of Mathematical Control and Information, 27(4):419, 2010.

[18] A. Megretski and A. Rantzer. System analysis via integral quadratic constraints. IEEE Transactions on Automatic Control, 42(6):819-830, Jun 1997.

[19] L. Mirkin. Some remarks on the use of time-varying delay to model sample-and-hold circuits. IEEE Transactions on Automatic Control, 52(6):11091112, June 2007.

[20] H. Omran, L. Hetel, M. Petreczky, J. P. Richard, and F. Lamnabhi-Lagarrigue. Stability analysis of some classes of input-affine nonlinear systems with aperiodic sampled-data control. Automatica, 70:266 - 274, 2016.

[21] J. Ploeg, N. van de Wouw, and H. Nijmeijer. Lp string stability of cascaded systems: Application to vehicle platooning. IEEE Transactions on Control Systems Technology, 22(2):786-793, March 2014.

[22] M.B.G. Posthumus - Cloosterman. Control over communication networks : modeling, analysis, and synthesis. PhD thesis, Department of Mechanical Engineering, TU Eindhoven, 2008.

[23] J. Thomas, C. Fiter, L. Hetel, N.v.d. Wouw, and J. P. Richard. Dissipativity-based framework for stability analysis of aperiodically sampled nonlinear systems with time-varying delay. Submitted, 2019.

[24] J. Thomas, L. Hetel, C. Fiter, N. van de Wouw, and J. Richard. $L_{2}$-stability criterion for systems with decentralized asynchronous controllers. In 2018 IEEE Conference on Decision and Control (CDC), pages 6638-6643, Dec 2018.

[25] Masashi Wakaiki, Kunihisa Okano, and João P. Hespanha. Stabilization of systems with asynchronous sensors and controllers. Automatica, 81:314 - 321, 2017.

[26] Wei Zhang, M. S. Branicky, and S. M. Phillips. Stability of networked control systems. IEEE Control Systems Magazine, 21(1):84-99, 2001.

[27] F. Xiao, Y. Shi, and W. Ren. Robustness analysis of asynchronous sampled-data multiagent networks with time-varying delays. IEEE Transactions on Automatic Control, 63(7):2145-2152, 2018.

\section{A Proof of Theorem 1}

From the definition of $\eta(t)$ in $(11)$, we have $\dot{\eta}(t)=\dot{z}(t)+$ $A_{c l} e^{A_{c l} t} x_{0}$. Substituting $\dot{z}(t)$ from (7),

$$
\begin{aligned}
\dot{\eta}(t) & =A_{c l} z(t)+B_{c l} u_{z}(t)+w(t)+A_{c l} e^{A_{c l} t} x_{0}, \\
& =A_{c l}\left(z(t)+e^{A_{c l} t} x_{0}\right)+B_{c l} u_{z}(t)+w(t) .
\end{aligned}
$$

Substituting $\eta$ as defined in (11), we have

$$
\dot{\eta}(t)=A_{c l} \eta(t)+B_{c l} u_{z}(t)+w(t),
$$

with $\eta(0)=x_{0}$, and $u_{z}(t)$ as given in (8). The system (A.2) has been shown in Figure 3.

1) For $t \in\left[0, a_{0}\right)$ : As per definition $(9)$, we have $e(t)=0$. Consequently, using the definition of $g(t)$ in $(12), u_{z}(t)=$ $g(t)=x_{i n i t}-\mu(t), \forall t \in\left[0, a_{0}\right)$. Hence, we have from (A.2)

$$
\dot{\eta}(t)=A_{c l} \eta(t)+B_{c l}\left(x_{i n i t}-\mu(t)\right)+w(t) .
$$

Note that the signal $\mu$ in (12), satisfies $\dot{\mu}(t)=A \mu(t)+$ $B_{c l} x_{i n i t}+w(t)$, where $B_{c l}=B K$ and $\mu(0)=x_{0}$. Therefore

$$
\begin{aligned}
\dot{\mu}(t) & =A \mu(t)+B_{c l} \mu(t)-B_{c l} \mu(t)+B_{c l} x_{i n i t}+w(t), \\
& =A_{c l} \mu(t)+B_{c l}\left(x_{i n i t}-\mu(t)\right)+w(t) .
\end{aligned}
$$

Comparing (A.3) and (A.4), since $\mu(0)=\eta(0)=x(0)=$ $x_{0}$, and $\dot{x}(t)=A x(t)+B K x_{i n i t}+w(t), \forall t \in\left[0, a_{0}\right)$, we have,

$$
\eta(t)=\mu(t)=x(t), \forall t \in\left[0, a_{0}\right) .
$$

2) For $t \geq a_{0}$ : From (12), we have $g(t)=0$, for all $t \geq a_{0}$. Therefore, from the definition of $u_{z}(t)$ in $(8)$ and $e(t)$ in (9), we have

$$
u_{z}(t)=e(t)=(\Delta y)(t), \forall t \geq a_{0} .
$$

From the interconnection (7)-(10), we have $y(t)=y_{z}(t)+$ $f(t)=A_{c l} z(t)+B_{c l} u_{z}(t)+w(t)+A_{c l} e^{A_{c l} t} x_{0}=A_{c l}(z(t)+$ $\left.e^{A_{c l} t} x_{0}\right)+B_{c l} u_{z}(t)+w(t)=A_{c l} \eta(t)+B_{c l} u_{z}(t)+w(t)$. Therefore, from (A.2), we can conclude $y(t)=\dot{\eta}(t)$ for all $t \geq 0$. Consequentially, we have from (A.6),

$$
u_{z}(t)=e(t)=(\Delta \dot{\eta})(t)=\eta\left(s_{k}\right)-\eta(t), \forall t \in\left[a_{k}, a_{k+1}\right)
$$

This transformation of capturing the sampling- and asynchrony-induced effects using an operator is based on similar works given in $[3,6,11,14,15,16,19]$. By substituting (A.7) in (A.2), we have for all $t \in\left[a_{k}, a_{k+1}\right)$,

$$
\begin{aligned}
\dot{\eta}(t) & =A_{c l} \eta(t)+B_{c l}\left(\eta\left(s_{k}\right)-\eta(t)\right)+w(t), \\
& =A \eta(t)+B K \eta(t)+B K \eta\left(s_{k}\right)-B K \eta(t)+w(t), \\
& =A \eta(t)+B K \eta\left(s_{k}\right)+w(t) .
\end{aligned}
$$


Comparing (A.8) with (2) and (3), and since $\eta\left(a_{0}\right)=$ $x\left(a_{0}\right)$ from (A.5), we can conclude that $\eta(t)=x(t), \forall t \geq$ $a_{0}$. Using this and (11), (A.5), we have $x(t)=\eta(t)=$ $z(t)+e^{A_{c l} t} x_{0}, \forall t \geq 0$.

\section{B Proof of Lemma 4}

Consider $e_{i}(t)$ defined using the operator $\Delta_{i}$ given in (38). We have, $e_{i}(t)=-\int_{s_{k}^{i}}^{t} y_{i}(\theta) d \theta, \forall t \in\left[a_{k}^{i}, a_{k+1}^{i}\right), k \in$ $\mathbb{N}$. By virtue of Jensen's inequality, we can state

$$
\begin{aligned}
e_{i}(t)^{T} R_{i} e_{i}(t) & =\left(\int_{s_{k}^{i}}^{t} y_{i}(\theta) d \theta\right)^{T} R_{i}\left(\int_{s_{k}^{i}}^{t} y_{i}(\theta) d \theta\right) \\
& \leq\left(t-s_{k}^{i}\right) \int_{s_{k}^{i}}^{t} y_{i}^{T}(\theta) R_{i} y_{i}(\theta) d \theta,
\end{aligned}
$$

and since $t \in\left[a_{k}^{i}, a_{k+1}^{i}\right)$, from (14), (15), we obtain $t-s_{k}^{i} \leq a_{k+1}^{i}-s_{k}^{i}=s_{k+1}^{i}+\eta_{k+1}^{i}-s_{k}^{i}=h_{k}^{i}+\eta_{k+1}^{i} \leq \bar{h}_{i}+\bar{\eta}_{i}$. Therefore, $e_{i}(t)^{T} R_{i} e_{i}(t) \leq\left(\bar{h}_{i}+\bar{\eta}_{i}\right) \int_{s_{k}^{i}}^{t} y_{i}^{T}(\theta) R_{i} y_{i}(\theta) d \theta$. Substituting $\theta=t+p$ and once again using the fact that $t \in\left[a_{k}^{i}, a_{k+1}^{i}\right)$, we have $e_{i}(t)^{T} R_{i} e_{i}(t) \leq$ $\left(\bar{h}_{i}+\bar{\eta}_{i}\right) \int_{-\left(\bar{h}_{i}+\bar{\eta}_{i}\right)}^{0} y_{i}^{T}(t+p) R_{i} y_{i}(t+p) d p$. Hence,

$$
\begin{aligned}
& \int_{a_{0}^{i}}^{\infty} e_{i}(t)^{T} R_{i} e_{i}(t) d t \leq \\
& \quad\left(\bar{h}_{i}+\bar{\eta}_{i}\right) \int_{a_{0}^{i}}^{\infty}\left(\int_{-\left(\bar{h}_{i}+\bar{\eta}_{i}\right)}^{0} y_{i}^{T}(t+p) R_{i} y_{i}(t+p) d p\right) d t \\
& \leq\left(\bar{h}_{i}+\bar{\eta}_{i}\right) \int_{-\left(\bar{h}_{i}+\bar{\eta}_{i}\right)}^{0}\left(\int_{a_{0}^{i}}^{\infty} y_{i}^{T}(t+p) R_{i} y_{i}(t+p) d t\right) d p,
\end{aligned}
$$

where $\theta=t+p$, implying $\theta \rightarrow \infty$ as $t \rightarrow \infty$ and $\theta \rightarrow a_{0}^{i}+p$ as $t \rightarrow a_{0}^{i}$. Since $a_{0}^{i} \geq \bar{h}_{i}+\bar{\eta}_{i}, p \in\left[-\left(\bar{h}_{i}+\bar{\eta}_{i}\right), 0\right]$ and the integrand of the inner integral is the positive term $y_{i}^{T}(t+p) R_{i} y_{i}(t+p)=y_{i}^{T}(\theta) R_{i} y_{i}(\theta)$, we can upper bound the aforementioned inequality by $\int_{a_{0}^{i}}^{\infty} e_{i}(t)^{T} R_{i} e_{i}(t) d t \leq$ $\left(\bar{h}_{i}+\bar{\eta}_{i}\right) \int_{-\left(\bar{h}_{i}+\bar{\eta}_{i}\right)}^{0}\left(\int_{0}^{\infty} y_{i}^{T}(\theta) R_{i} y_{i}(\theta) d \theta\right) d p$. As per the definition of $e_{i}(t)$ in $(38)$, since $e_{i}(t)=0, \forall t \in\left[0, a_{0}^{i}\right)$, we have $\int_{0}^{\infty} e_{i}(t)^{T} R_{i} e_{i}(t) d t=\int_{a_{0}^{i}}^{\infty} e_{i}(t)^{T} R_{i} e_{i}(t) d t \leq$ $\gamma_{i}^{2} \int_{0}^{\infty} y_{i}^{T}(t) R_{i} y_{i}(t) d t$, with $\gamma_{i}=\bar{h}_{i}+\bar{\eta}_{i}$. Hence, we have

$$
\int_{0}^{\infty}\left[\begin{array}{l}
y_{i}(t) \\
e_{i}(t)
\end{array}\right]^{T}\left[\begin{array}{cc}
\gamma_{i}^{2} R_{i} & 0 \\
0 & -R_{i}
\end{array}\right]\left[\begin{array}{l}
y_{i}(t) \\
e_{i}(t)
\end{array}\right] d t \geq 0
$$

$\gamma_{i}$ is essentially the upper bound on the $\mathcal{L}_{2}$ induced norm of the operator $\Delta_{i}$. Considering the integral quadratic constraint (B.3) for all $i \in\{1,2, \ldots, M\}$, i.e., for the operator $\boldsymbol{\Delta}$, we have $\int_{0}^{\infty}\left[\begin{array}{l}y(t) \\ e(t)\end{array}\right]^{T}\left[\begin{array}{cc}S & 0 \\ 0 & -R\end{array}\right]\left[\begin{array}{l}y(t) \\ e(t)\end{array}\right] d t \geq 0$, where $e(t)=\left[e_{1}^{T}(t), e_{2}^{T}(t), \ldots, e_{M}^{T}(t)\right]^{T}$, $y(t)=\left[y_{1}^{T}(t), y_{2}^{T}(t), \ldots, y_{M}^{T}(t)\right]^{T}$, and $S=\operatorname{diag}\left(\gamma_{1}^{2} R_{1}, \gamma_{2}^{2} R_{2}, \ldots, \gamma_{M}^{2} R_{M}\right)$.

\section{Proof of Lemma 7}

Consider the term

$$
e_{i}^{s^{\star}}(t)=\left(\Delta_{i}^{s^{\star}} y_{i}\right)(t), \forall t \geq 0
$$

where $\left(\Delta_{i}^{s^{\star}} y_{i}\right)(t)=\left\{\begin{array}{l}0, \forall t \in\left[0, s_{0}^{i}\right), \\ -\int_{s_{k}^{i}}^{t} y_{i}(\theta) d \theta, \forall t \in\left[s_{k}^{i}, s_{k+1}^{i}\right), k \in \mathbb{N},\end{array}\right.$ where $y_{i}(t)=\dot{x}_{i}(t), \forall t \geq 0$. Therefore, we have $e_{i}^{s^{\star}}(t)=\left\{\begin{array}{l}0, \forall t \in\left[0, s_{0}^{i}\right), \\ x_{i}\left(s_{k}^{i}\right)-x_{i}(t), \forall t \in\left[s_{k}^{i}, s_{k+1}^{i}\right), k \in \mathbb{N} .\end{array}\right.$

Consider the expression $\sum_{k=0}^{\infty} \int_{s_{k}^{i}}^{s_{k+1}^{i}} e_{i}^{s^{\star} T}(t) R_{i}^{s} e_{i}^{s^{\star}}(t) d t$, where $R_{i}^{s}>0$ is a scaling matrix. By virtue of the Wirtinger inequality [17], we can state

$\sum_{k=0}^{\infty} \int_{s_{k}^{i}}^{s_{k+1}^{i}} e_{i}^{s^{\star T}}(t) R_{i}^{s} e_{i}^{s^{\star}}(t) d t \leq$

$\sum_{k=0}^{\infty} \frac{4\left(s_{k+1}^{i}-s_{k}^{i}\right)^{2}}{\pi^{2}} \int_{s_{k}^{i}}^{s_{k+1}^{i}} \frac{d}{d t}\left(e_{i}^{s^{\star T}}(t)\right) R_{i}^{s} \frac{d}{d t}\left(e_{i}^{s^{\star}}(t)\right) d t$. Since $s_{k+1}^{i}-s_{k}^{i} \leq \bar{h}_{i}, \forall k \in \mathbb{N}$, we have

$\sum_{k=0}^{\infty} \int_{s_{k}^{i}}^{s_{k+1}^{i}} e_{i}^{s^{\star} T}(t) R_{i}^{s} e_{i}^{s^{\star}}(t) d t$

$\leq \frac{4 \bar{h}_{i}^{2}}{\pi^{2}} \int_{s_{k}^{i}}^{s_{k+1}^{i}} \frac{d}{d t}\left(e_{i}^{s^{\star} T}(t)\right) R_{i}^{s} \frac{d}{d t}\left(e_{i}^{s^{\star}}(t)\right) d t$

$=\frac{4 \bar{h}_{i}^{2}}{\pi^{2}} \sum_{k=0}^{\infty} \int_{s_{k}^{i}}^{s_{k+1}^{i}} \frac{d}{d t}\left(\Delta_{i}^{s^{\star}} y_{i}\right)^{T}(t) R_{i}^{s} \frac{d}{d t}\left(\Delta_{i}^{s^{\star}} y_{i}\right)(t) d t$

$=\frac{4 \bar{h}_{i}^{2}}{\pi^{2}} \sum_{k=0}^{\infty} \int_{s_{k}^{i}}^{s_{k+1}^{i}} y_{i}(t)^{T} R_{i}^{s} y_{i}(t) d t$

$\leq \frac{4 \bar{h}_{i}^{2}}{\pi^{2}} \int_{0}^{\infty} y_{i}(t)^{T} R_{i}^{s} y_{i}(t) d t$. We have from (C.1) that $e_{i}^{s^{\star}}(t)=0$ for all $t \leq s_{0}^{i}$, implying $\int_{0}^{\infty} e_{i}^{s^{\star T}}(t) R_{i}^{s} e_{i}^{s^{\star}}(t) d t=$ $\int_{s_{0}^{i}}^{\infty} e_{i}^{s^{\star} T}(t) R_{i}^{s} e_{i}^{s^{\star}}(t) d t \leq \frac{4 \bar{h}_{i}^{2}}{\pi^{2}} \int_{0}^{\infty} y_{i}(t)^{T} R_{i}^{s} y_{i}(t) d t$. From (71) and (C.1), we have that $e_{i}^{s}(t)=e_{i}^{s^{\star}}(t)$ for all $t \geq a_{0}^{i}$, and $e_{i}^{s}(t)=0$ for all $t \leq a_{0}^{i}$, thereby implying $\int_{0}^{\infty} e_{i}^{s^{T}}(t) R_{i}^{s} e_{i}^{s}(t) d t \leq \int_{0}^{\infty} e_{i}^{s^{\star T}}(t) R_{i}^{s} e_{i}^{s^{\star}}(t) d t \leq$ $\frac{4 \bar{h}_{i}^{2}}{\pi^{2}} \int_{0}^{\infty} y_{i}(t)^{T} R_{i}^{s} y_{i}(t) d t$. Consequently, for all $i=$ $\{1,2, \ldots, M\}$, we have

$$
\int_{0}^{\infty}\left[\begin{array}{l}
y_{i}(t) \\
e_{i}^{s}(t)
\end{array}\right]^{T}\left[\begin{array}{cc}
\gamma_{i}^{s} R_{i}^{s} & 0 \\
0 & -R_{i}^{s}
\end{array}\right]\left[\begin{array}{c}
y(t) \\
e_{i}^{s}(t)
\end{array}\right] d t \geq 0
$$

where $\gamma_{i}^{s}=\frac{2 \bar{h}_{i}}{\pi}$. Considering the integral quadratic constraint (C.2) for all $i \in\{1,2, \ldots, M\}$, i.e., for the operator $\boldsymbol{\Delta}^{\mathbf{s}}$, we have $\int_{0}^{\infty}\left[\begin{array}{c}y(t) \\ e_{s}(t)\end{array}\right]^{T}\left[\begin{array}{cc}S_{s} & 0 \\ 0 & -R_{s}\end{array}\right]\left[\begin{array}{c}y(t) \\ e_{s}(t)\end{array}\right] d t \geq 0$, where

$$
\begin{aligned}
e_{s}(t) & =\left[e_{1}^{s T}(t), e_{2}^{s^{T}}(t), \ldots, e_{M}^{s}{ }^{T}(t)\right]^{T}, \\
y(t) & =\left[y_{1}^{T}(t), y_{2}^{T}(t), \ldots, y_{M}^{T}(t)\right]^{T}, \\
S_{s} & =\operatorname{diag}\left(\left(\gamma_{1}^{s}\right)^{2} R_{1}^{s},\left(\gamma_{2}^{s}\right)^{2} R_{2}^{s}, \ldots,\left(\gamma_{M}^{s}\right)^{2} R_{M}^{s}\right) .
\end{aligned}
$$

